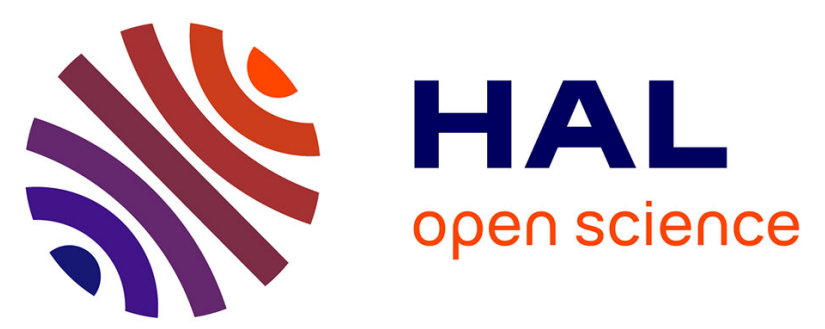

\title{
Rice local phase angle study for a delamination problem between a shape memory alloy and an elastic material
}

\author{
Mohamed Rachid Laydi, Christian Lexcellent
}

\section{To cite this version:}

Mohamed Rachid Laydi, Christian Lexcellent. Rice local phase angle study for a delamination problem between a shape memory alloy and an elastic material. Archive for Rational Mechanics and Analysis, 2012, 204 (3), pp.977 - 1007. 10.1007/s00205-012-0495-6 . hal-02300117

\section{HAL Id: hal-02300117 https://hal.science/hal-02300117}

Submitted on 19 Nov 2019

HAL is a multi-disciplinary open access archive for the deposit and dissemination of scientific research documents, whether they are published or not. The documents may come from teaching and research institutions in France or abroad, or from public or private research centers.
L'archive ouverte pluridisciplinaire HAL, est destinée au dépôt et à la diffusion de documents scientifiques de niveau recherche, publiés ou non, émanant des établissements d'enseignement et de recherche français ou étrangers, des laboratoires publics ou privés. 


\title{
Rice Local Phase Angle Study for a Delamination Problem Between a Shape Memory Alloy and an Elastic Material
}

\author{
Mohamed Rachid LAYdi \& CHRISTIAN LEXCELLENT
}

\begin{abstract}
The present study is an extension of a recent paper of Freed et al. ( $\mathrm{J}$ Mech Phys Solids 56:3003-3020, 2008). The final aim is to describe the transformation toughening behavior of a static crack along an interface between a shape memory alloy (SMA) and a linear elastic isotropic material. With an SMA as an equivalent Huber-Von Mises stress model (hypothesis of symmetric behavior between tension and compression), Freed et al. determine the initiation (ending) phase transformation yield surfaces in terms of the local phase angle introduced by Rice et al. (Metal ceramic interfaces, Pergamon Press, New York, pp 269-294, 1990). In this paper we give the general framework to determine this angle for a model integrating the asymmetry between tension and compression (experimentally measured: Vacher and Lexcellent in Proc ICM 6:231-236, 1991; Orgéas and Favier in Acta Mater 46(15):5579-5591, 2000), the Huber-Von Mises model being only a particular case. We demonstrate the local phase angle existence in an appropriate framing domain and give a sufficient hypothesis for its uniqueness and an algorithm to obtain it. Estimates are obtained in terms of physical quantities such as the Young modulus ratio, the bimaterial Poisson modulus values and also the choice of the yield loading functions. Finally, we illustrate this theoretical study by an application linking the asymmetry intensity on the width and the shape on predicted phase transformation surfaces and by a comparison with the symmetric case.
\end{abstract}

\section{Introduction}

Following the work of RICE et al. [1], we investigate the elastic-brittle fracture theory for cracks between dissimilar solids: a lower and an upper (see an illustration on Fig. 1). In our case, the upper layer is constituted by a shape memory alloy (SMA) and the lower by an isotropic elastic solid. 


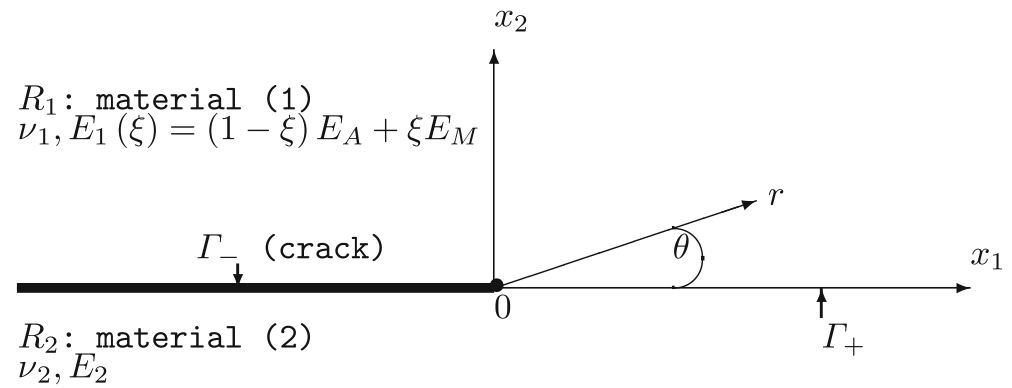

Fig. 1. Region near crack tip along bimaterial interface

Depending on the mechanical loading intensity, the SMA, initially in its austenitic state (with stress free state), can be subjected to a phase transformation (austenite-martensite). In this case its behavior is considered as pseudoelastic.

If the material is in its martensitic state (and stress free), a reorientation of martensite platelets called pseudoplasticity can be observed under loading. Specifically, the stresses at the neighborhood of a crack tip are square-root singular, as described by Yi and GAO [2] and FreED and BANKS-SiLls [3], implying that they are unbounded. As a consequence, a stress induced phase transformation (or a detwinning of martensite platelets) appears in the neighborhood of a crack tip at the early beginning of the load increment (FREED et al. [4]). Hence a disturbance is observed in this region.

The first aim of the present investigation is to compute the phase transformation surfaces corresponding to the phase transformation beginning (ending) around the crack tip.

Under the assumption of symmetry between tension and compression for pseudoelastic SMA behavior, FREED et al. [4] constructed these surfaces using a Huber-Von Mises equivalent stress SMA model as performed by PANOSKALTIS et al. [5].

In the present paper, we want to take into account the obvious asymmetry between tension and compression as it was measured by VACHER and LEXCELLENT [6], OrgÉAS and FAVIER [7]. We want to examine its impact on the shape and the width of the predicted phase transformation surfaces.

Moreover, particular attention must be paid to the "local phase angle" of the field as introduced by RICE et al. [1]. This local phase angle $\widehat{\psi}$ depends on the applied phase angle $\psi$ and also on the respective material elastic characteristics of the SMA material (1) and material (2) through an oscillatory parameter $\varepsilon$ to be defined. A particular investigation will be devoted to determine $\widehat{\psi}$ as a solution of a nonlinear equation, its existence and uniqueness and, finally, its bounds.

Particular local phase angles $\widehat{\psi}=\psi_{s}$ and $\widehat{\psi}=\psi_{f}(s$ for start and $f$ for finish phase transformation) will be obtained for the determination of the two surfaces. We will examine their changes as a function of the asymmetry intensity between tension and compression. 


\section{Stress Tensor and Local Phase Angle}

In this section, we give the form of the stress fields from linear elastic fracture mechanics (LEFM) theory for cracks along an interface between dissimilar materials (see Fig. 1). The case of joined isotropic materials was treated by RICE et al. [1], and Suo [8].

In fact, it is easy to use the LEFM theory because RICE et al. [1] provide the stress state for a bimaterial. Evidently, the stress field around the crack tip is unbounded. But loading mode I experiments performed on thin sheets of nitinol by DALY et al. [9] reveal a small-scale zone of phase transformation around the crack tip. Our study is restricted to loading. In this case, the pseudoelastic shape memory alloy behavior can be considered to be similar to the elasto-plastic behavior as investigated by RicE et al. [10]. In this spirit, the austenitic behavior, at least in its polycrystalline state, can be considered as an elastic isotropic behavior. The paper [10] reviews the fundamentals of elasto-plastic finite element analysis and its applications to the mechanics of crack opening and growth in ductile solids. One must note that SMAs are more brittle than ductile (10\% of maximum pseudoelastic strain before fracture).

\subsection{Notations}

We will use cartesian coordinates $x_{1}, x_{2}, x_{3}$ and cylindrical coordinates $r, \theta, z$ with the usual rules $x_{3}=z, x_{1}+i x_{2} \equiv r \exp (i \theta) \in \mathbb{C}$.

Let us investigate a horizontal crack in a bimaterial made of a pseudoelastic SMA material (1) and an elastic material (2). As shown on Fig. 1, the crack is located at the interface between the two materials in the plane $\left(x_{1}, x_{3}\right)$ in the side $x_{1}<0$.

In every case, the linear elastic fracture mechanics (LEFM) theory is used. Our purpose is restricted to plane conditions; that is, a plane stress denoted by $\mathrm{CP}$ and plane strains denoted by DP.

Let $R_{1} \equiv\left\{\left(x_{1}, x_{2}\right) \in \mathbb{R}^{2} \mid x_{2}>0\right\}$ and $R_{2} \equiv\left\{\left(x_{1}, x_{2}\right) \in \mathbb{R}^{2} \mid x_{2}<0\right\}$ the respective domains of material (1) and material (2) in $\left.\mathbb{R}^{2}, \Gamma_{-} \equiv\right]-\infty, 0[\times\{0\}$ a part of the interface associated to the crack, $\left.\Gamma_{+} \equiv\right] 0,+\infty[\times\{0\}$ the remaining part of the interface without the origin and $R \equiv R_{1} \cup R_{2} \cup \Gamma_{+}$. It is clear that $R$ is also a domain (that is an open connected subset) of $\mathbb{R}^{2}$. We will often identify $R$ with the subset of complex numbers

$$
\left\{z \in \mathbb{C} \mid z \equiv x_{1}+i x_{2}, \forall\left(x_{1}, x_{2}\right) \in R\right\} .
$$

In this study, the intensity of loading is such that material (2) behaves as an elastic isotropic body with Young modulus $E_{2}$ and Poisson ratio $\nu_{2}$. For material (1) the Poisson ratio $v_{1}$ is the same whatever the phase state. With low stress, material (1) is austenitic with Young modulus $E_{1}=E_{A}$ and with higher stress, martensitic with Young modulus $E_{1}=E_{M}$. For an intermediate state, that is, biphased with $\xi$ volume fraction of martensite ( $1-\xi$ volume fraction of austenite), the mixing rule delivers the equivalent Young modulus

$$
E_{1}(\xi) \equiv(1-\xi) E_{A}+\xi E_{M}, \quad 0 \leqq \xi \leqq 1 .
$$


In a classical way, the phase state (austenitic, martensitic or biphased) is characterized by a convex function $\mathcal{G}$ in the stress space $\sigma$, called loading function, such that

$$
0 \leqq \mathcal{G}(\sigma)<\sigma_{A} \quad \text { in } R_{1} \cup \Gamma_{+}
$$

corresponds to elastic domain of austenite, where $\sigma_{A}$ is the yield stress of martensite initiation $(\xi=0)$. If $\sigma_{M}$ is the yield stress of martensite ending $(\xi=1)$, the two phas domain is defined by

$$
\sigma_{A} \leqq \mathcal{G}(\sigma) \leqq \sigma_{M} \quad \text { in } R_{1} \cup \Gamma_{+} .
$$

A yield surface of equifraction of martensite can be described by

$$
\mathcal{G}(\sigma)=\sigma_{c}, \quad \text { in } R_{1} \cup \Gamma_{+},
$$

where

$$
\sigma_{c}(\xi) \equiv(1-\xi) \sigma_{A}+\xi \sigma_{M}, \quad 0<\sigma_{A} \leqq \sigma_{M}, \quad 0 \leqq \xi \leqq 1
$$

Note that the loading function $\mathcal{G}$ is related to material (1) only. Moreover, $\sigma_{\mid R_{1}}$ and $\sigma_{\mid R_{2}}$ (the respective restrictions of the stress tensor $\sigma$ on the open sets $R_{1}$ and $R_{2}$ ) are continuous on $R_{1}$ and $R_{2}$, but $\sigma$ may have a discontinuity of the first kind on $\Gamma_{+}$. In all cases, the trace of $\sigma$ on $\Gamma_{+}$appearing in (2)-(4) refers to the trace of $\sigma_{\mid R_{1}}$ on $\Gamma_{+}$.

Remark 1. Practically,

$$
\left\{\begin{array}{l}
\sigma_{A} \equiv C_{M}\left(T-M_{\mathrm{S}}\right) \\
\sigma_{M} \equiv C_{M}\left(T-M_{\mathrm{f}}\right)
\end{array},\right.
$$

where $T$ is the test temperature, $M_{\mathrm{S}}\left(M_{\mathrm{f}}\right)$ is the martensite start (finish) temperature at stress free state and $C_{M}$ is the slope of the one-dimensional stress-temperature linear relation in the Clausius Clapeyron diagram under the following conditions:

$$
T>M_{\mathrm{S}}>M_{\mathrm{f}} \text { and } C_{M}>0 .
$$

An another crucial parameter, called in our study $\varepsilon$, represents the difference between elastic properties of materials (1) and (2). This parameter, named "oscillatory parameter" by DUNDURS [11], is given by

$$
\varepsilon \equiv \frac{1}{2 \pi} \ln \left(\frac{1-\beta}{1+\beta}\right),
$$

where $\beta$ is the second Dundurs parameter [11]. For an interface between two isotropic linear elastic materials, $\beta$ takes the following shape

$$
\beta \equiv \frac{\mu_{1}\left(\kappa_{2}-1\right)-\mu_{2}\left(\kappa_{1}-1\right)}{\mu_{1}\left(\kappa_{2}+1\right)+\mu_{2}\left(\kappa_{1}+1\right)},
$$

where

$$
\mu_{\alpha} \equiv \frac{E_{\alpha}}{2\left(1+v_{\alpha}\right)}>0
$$


is the shear and $\kappa_{\alpha}$ is the volume expansion coefficient

$$
\kappa_{\alpha} \equiv\left\{\begin{array}{ll}
\frac{3-v_{\alpha}}{1+v_{\alpha}} & \mathrm{CP} \\
3-4 v_{\alpha} & \text { DP }
\end{array} .\right.
$$

Note that the parameter $\varepsilon$ is equal to zero when the two materials are the same. Note also that the parameter $\varepsilon$ changes of sign if one exchanges the two materials. Its value moves from (see (11))

$$
|\varepsilon|<\frac{\ln 3}{2 \pi}<0.175
$$

Remark 2. One can check (see Lemma 2 in Appendix B) that

$$
|\beta|<\frac{1}{2}
$$

so that the parameter $\varepsilon$ (see (6)) is well defined.

In the particular case $v_{1}=v_{2}=v$, the expression of $\beta$ is simpler and reads

$$
\beta=\frac{1}{2} \widetilde{e}\left\{\begin{array}{l}
1-v \mathrm{CP} \\
\frac{1-2 v}{1-v} \mathrm{DP}
\end{array} \text { with } \widetilde{e} \equiv \frac{1-e}{1+e}, e \equiv \frac{E_{2}}{E_{1}} .\right.
$$

Remark 3. In FreED et al. [4] (this has been confirmed by a private communication), instead of (1), one uses the rule

$$
\varepsilon(\xi) \equiv(1-\xi) \varepsilon_{A}+\xi \varepsilon_{M}, 0 \leqq \xi \leqq 1,
$$

where $\varepsilon_{A}$ and $\varepsilon_{M}$ denote the values of $\varepsilon$ obtained by (6), respectively, with $E_{1}=E_{A}$ and with $E_{1}=E_{M}$. In general the two rules are different except, obviously, for $\xi=0$ or $\xi=1$. If $0<\xi<1$, one deduces $\beta$ by inverting (6), that is,

$$
\beta \equiv \tanh (-\varepsilon \pi) .
$$

Inequality (11) remains true because (10) remains true for (13). This is not the case for Equality (12), which is no longer true.

Although the difference between the two rules has a physical relevance, our mathematical results are the same except (possibly) for Proposition 3, which should be treated without referring to (12).

\subsection{Setting of the Problem}

For simplicity of notation, the index $\alpha$ refers to the two cases $\alpha=1,2$. The mathematical problem can be stated as:

Let $\mathcal{G}$ be a suitable convex function (its properties will be made precise later, see (35)) and let $\xi$ be a fixed internal parameter in $[0,1]$. Given

$$
\left.v_{\alpha} \in\right] 0, \frac{1}{2}\left[, E_{\alpha}>0, \sigma_{c}>0 \text { and } K \in \mathbb{C}^{*},\right.
$$


find an angle

$$
\widehat{\psi} \equiv \arctan \left(\frac{\sigma_{12}}{\sigma_{22}}\right) \text { on } \Gamma_{+}
$$

such that

$$
\mathcal{G}\left(\sigma_{\mid R_{1}}\right)=\sigma_{c} \text { on } \Gamma_{+},
$$

where the stress tensor is a function of $\left(x_{1}, x_{2}\right) \in R$ such that

$$
\boldsymbol{\sigma} \equiv\left(\begin{array}{ccc}
\sigma_{11} & \sigma_{12} & 0 \\
\sigma_{21} & \sigma_{22} & 0 \\
0 & 0 & \sigma_{33}
\end{array}\right)
$$

with

$$
\sigma_{33 \mid R_{\alpha}} \equiv\left\{\begin{array}{ll}
0 & \mathrm{CP} \\
v_{\alpha}\left(\sigma_{11}+\sigma_{22}\right) & \mathrm{DP}
\end{array} .\right.
$$

The stress $\sigma_{j k}$, the strain $\varepsilon_{j k}$ and the displacements $u_{1}, u_{2}$ are linked by the classical Hooke equations

$$
\left\{\begin{array}{ll}
\varepsilon_{j k} \equiv \frac{1+v_{\alpha}}{E_{\alpha}} \sigma_{j k}-\frac{v_{\alpha}}{E_{\alpha}}\left(\sigma_{11}+\sigma_{22}+\sigma_{33}\right) \delta_{j k} & \text { in } R_{\alpha} \\
\varepsilon_{j k} \equiv \frac{1}{2}\left(\frac{\partial u_{j}}{\partial x_{k}}+\frac{\partial u_{k}}{\partial x_{j}}\right) & \text { in } R
\end{array},\right.
$$

where $j, k=1,2$ and $\delta_{j k}$ is the Kronecker symbol.

Besides the compatibility relations induced by (19), the functions $\sigma_{j k}$ satisfy, in addition,

$$
\begin{aligned}
& \left\{\begin{array}{ll}
\frac{\partial \sigma_{11}}{\partial x_{1}}+\frac{\partial \sigma_{12}}{\partial x_{2}}=0 & \text { in } R \\
\frac{\partial \sigma_{12}}{\partial x_{1}}+\frac{\partial \sigma_{22}}{\partial x_{2}}=0 & \text { in } R \\
\sigma_{12}=\sigma_{22}=0 & \text { on } \Gamma_{-}
\end{array},\right. \\
& \sigma_{22}+i \sigma_{12}=\frac{K r^{i \varepsilon}}{\sqrt{2 \pi r}} \text { on } \Gamma_{+} \text {, }
\end{aligned}
$$

and

$$
\lim _{r \rightarrow+\infty} \sigma_{11}=0
$$

The angle $\widehat{\psi}$ represents the local modal mixity on the interface $\Gamma_{+}$. The system (20) expresses (in absence of volumic forces) the balance equations in $R$ with the boundary condition of zero normal stresses. The interest of Equations (21)-(22) is to select the purely singular solutions only, the ones which provide the asymptotic behavior of stress in the vicinity of the origin (see Theorem 1).

Condition (21) characterizes the stress intensity factor $K$ and Condition (22) fixes the additive constant in the determination of $\sigma_{11}$. 
Remark 4. In our model the problems concerning $\widehat{\psi}$ and $\sigma$ are not coupled. The tensor $\sigma$ will be uniquely determined from Equations (17)-(22); the explicit expression of $\sigma$ will be given in Theorem 1. We will formulate later Equations (15)-(16) as a fixed point problem for $\widehat{\psi}$ (see Corollary 1 ).

As we will show later, this decoupling between the transformation surface equation and the balance equation is particularly due to the use of Hooke's law.

Of course, the nonlinearity of the pseudoelastic SMA does not allow, in general, the explicit computation of $\sigma$. Roughly, the strain tensor can be split as

$$
\varepsilon \equiv \varepsilon^{e}+\varepsilon^{t r},
$$

with $\varepsilon^{e}$ given in (19), and

$$
\varepsilon^{t r} \equiv \gamma \xi \frac{\partial \mathcal{G}}{\partial \boldsymbol{\sigma}}, \quad \xi \equiv \xi(\sigma, T) \in[0,1],
$$

where $\gamma$ is the phase transformation strain for pure shearing. Its detailed formulation can be found in LEXCELLENT et al. [12].

For a review of more general SMA models, we refer the reader to BERNARDINI and PENCE [13] and the references therein.

Remark 5. The boundaries of the transformation zones depend upon the near tip and far field stress intensity factors called, respectively, $K^{\text {tip }}$ and $K^{\text {app }}$. In the spirit of EvAns [14], the boundaries may be described by stress intensity factors which are an average of the far field and near tip ones, namely

$$
K \equiv K_{1}+i K_{2}=\frac{1}{2}\left(\left(K_{1}^{\mathrm{app}}+K_{1}^{\mathrm{tip}}\right)+i\left(K_{2}^{\mathrm{app}}+K_{2}^{\mathrm{tip}}\right)\right) .
$$

The complex $K$, given by RICE et al. [1], has the generic form

$$
K \equiv Y S \sqrt{\widehat{L}} \widehat{L}^{-i \varepsilon} \exp (i \psi)
$$

where $S$ is a representative magnitude of the stress applied to load the specimen, $\widehat{L}>0$ a characteristic length (for example crack length, layer thickness), $Y$ a dimensionless real positive quantity and $\psi$ by definition is the phase angle of $K \widehat{L}^{i \varepsilon}$. Therefore, $\psi$ is a measure of the relation between the shear and the normal stress component along the interface at a distance $\widehat{L}$.

From (23) and (21), we then have

$$
\widehat{\psi}(r, \varepsilon)=\psi+\varepsilon \ln \left(\frac{r}{\widehat{L}}\right) \text { on } \Gamma_{+}, \quad(\psi \text { and } \widehat{L} \text { being fixed }) .
$$

Note that, due to the introduction of $\widehat{L}$, the value of $\widehat{\psi}$ is independent of the choice of the units. However, it depends on $r$, so the tensile and in-plane modes (mode I and II) are fundamentally inseparable.

The variable quantity $\widehat{\psi}$ is called by RICE et al. [1] the "local phase angle" of the field. 


\subsection{Elastic Fields for Interface Crack}

In this section, we establish the explicit expression of tensor $\sigma$, the one which determines the local phase angle $\widehat{\psi}$ according to (15). We will check that $\sigma$ is nothing but the tensor already given by RICE [1] in a cylindrical coordinate system (see Appendix A).

Theorem 1. We assume (14). Then, Problem (17)-(22) admits a unique solution $\sigma$ such that

$$
\sigma=\frac{1}{\sqrt{2 \pi r}}\left(\operatorname{Re}\left(K r^{i \varepsilon}\right) \Sigma^{I}+\operatorname{Im}\left(K r^{i \varepsilon}\right) \Sigma^{I I}\right),
$$

where $\Sigma^{I}(\theta, \varepsilon, \bar{v})$ and $\Sigma^{I I}(\theta, \varepsilon, \bar{v})$ are given by

$$
\left\{\begin{aligned}
& \Sigma_{22}^{I}= \frac{\Upsilon_{\alpha}}{2 \cosh (\pi \varepsilon)}\left(\cos \frac{\theta}{2}+\sin \frac{3 \theta}{2} \sin \theta+\Upsilon_{\alpha}^{-2} \cos \frac{\theta}{2}-2 \varepsilon \cos \frac{3 \theta}{2} \sin \theta\right) \\
& \Sigma_{11}^{I}=\frac{2 \Upsilon_{\alpha}}{\cosh (\pi \varepsilon)} \cos \frac{\theta}{2}-\Sigma_{22}^{I} \\
& \Sigma_{12}^{I}=\frac{\Upsilon_{\alpha}}{2 \cosh (\pi \varepsilon)}\left(\sin \frac{3 \theta}{2} \cos \theta-\Upsilon_{\alpha}^{-2} \sin \frac{\theta}{2}+2 \varepsilon \sin \frac{3 \theta}{2} \sin \theta\right) \\
& \Sigma_{22}^{I I}=\frac{\Upsilon_{\alpha}}{2 \cosh (\pi \varepsilon)}\left(\cos \frac{3 \theta}{2} \sin \theta-\sin \frac{\theta}{2}+\Upsilon_{\alpha}^{-2} \sin \frac{\theta}{2}+2 \varepsilon \sin \frac{3 \theta}{2} \sin \theta\right) \\
& \Sigma_{11}^{I I}=-\frac{2 \Upsilon_{\alpha}}{\cosh (\pi \varepsilon)} \sin \frac{\theta}{2}-\Sigma_{22}^{I I} \\
& \Sigma_{12}^{I I}=\frac{\Upsilon_{\alpha}}{2 \cosh (\pi \varepsilon)}\left(\cos \frac{3 \theta}{2} \cos \theta+\Upsilon_{\alpha}^{-2} \cos \frac{\theta}{2}+2 \varepsilon \cos \frac{3 \theta}{2} \sin \theta\right) \\
& \quad \Sigma_{33}^{I}=\bar{v}\left(\Sigma_{11}^{I}+\Sigma_{22}^{I}\right), \Sigma_{33}^{I I}=\bar{v}\left(\Sigma_{11}^{I I}+\Sigma_{22}^{I I}\right)
\end{aligned}\right.
$$

where

$$
\Upsilon_{\alpha}=\exp \left(\varepsilon\left(\pi_{\alpha}+\theta\right)\right), \pi_{\alpha} \equiv(-1)^{\alpha} \pi
$$

and "equivalent Poisson coefficient" $\bar{v}$ is defined by

$$
\bar{v}_{\mid R_{\alpha}} \equiv\left\{\begin{array}{ll}
0 & \mathrm{CP} \\
v_{\alpha} & \mathrm{DP}
\end{array}, v_{\alpha} \in\right] 0, \frac{1}{2}[.
$$

Proof. According to the complex potentials method of Kolossov-Muskhelishvili, $\widehat{\sigma}$, the general solution to (17)-(20), is given by (see RICE [15])

$$
\left\{\begin{array}{l}
\widehat{\sigma}_{11}+\widehat{\sigma}_{22}=2\left(\frac{\mathrm{d} \Phi_{\alpha}}{\mathrm{d} z}+\frac{\overline{\mathrm{d} \Phi_{\alpha}}}{\mathrm{d} z}\right) \\
\widehat{\sigma}_{22}-\widehat{\sigma}_{11}+2 i \widehat{\sigma}_{12}=2\left((\bar{z}-z) \frac{\mathrm{d}^{2} \Phi_{\alpha}}{\mathrm{d} z^{2}}-\frac{\mathrm{d} \Phi_{\alpha}}{\mathrm{d} z}+\frac{\mathrm{d} \Omega_{\alpha}}{\mathrm{d} z}\right)
\end{array} \text { in } R_{\alpha},\right.
$$

$\Phi_{\alpha}$ and $\Omega_{\alpha}$ being complex functions defined on $R_{\alpha}$ by

$$
\left\{\begin{array}{c}
\frac{\mathrm{d} \Phi_{\alpha}}{\mathrm{d} z} \equiv \exp \left(\pi_{\alpha} \varepsilon\right) z^{-\frac{1}{2}-i \varepsilon} f+a_{\alpha} g \\
\frac{\mathrm{d} \Omega_{\alpha}}{\mathrm{d} z} \equiv \exp \left(-\pi_{\alpha} \varepsilon\right) z^{-\frac{1}{2}+i \varepsilon} \bar{f}-a_{\alpha} \bar{g}
\end{array},\right.
$$


where $z \equiv x_{1}+i x_{2}, a_{1} \equiv \frac{2 c_{2}}{c_{1}+c_{2}}, a_{2} \equiv \frac{2 c_{1}}{c_{1}+c_{2}}, c_{\alpha} \equiv \frac{\kappa_{\alpha}+1}{\mu_{\alpha}}, f$ and $g$ are analytic throughout $R$ and the overbar denotes complex conjugate. By summation in (29) we get from (30)

$$
\widehat{\sigma}_{22}+i \widehat{\sigma}_{12}=2 r^{-\frac{1}{2}+i \varepsilon} \cosh (\pi \varepsilon) \bar{f} \text { on } \Gamma_{+} .
$$

It follows that $\widehat{\sigma}$ is also a solution to (21) if and only if

$$
f(z)=\frac{\bar{K}}{2 \sqrt{2 \pi} \cosh (\pi \varepsilon)} \text { in } R .
$$

Indeed, the equality on $\Gamma_{+}$follows by comparison and extends to the Connex set $R$ by analyticity of $f$.

By choosing $g \equiv 0$ in (30), function $f$ being fixed by (32), we obtain the tensor $\sigma$ (25) which trivially satisfies Condition (22). Thus, $\sigma$ is a particular solution to (17)-(22).

Uniqueness: The tensor "difference" $\widetilde{\sigma} \equiv \widehat{\sigma}-\sigma$ is solution to (17)-(21) such that

$$
\left\{\begin{array}{ll}
\widetilde{\sigma}_{11}+\widetilde{\sigma}_{22}=2 a_{\alpha}(g+\bar{g}) & \text { in } R_{\alpha} \\
\widetilde{\sigma}_{22}+i \widetilde{\sigma}_{12}=a_{\alpha}(\bar{z}-z) \frac{\mathrm{d} g}{\mathrm{~d} z} & \text { in } R_{\alpha}
\end{array} .\right.
$$

In terms of the real part $P \equiv \operatorname{Re}(g)$, we have

$$
\left\{\begin{array}{l}
\widetilde{\sigma}_{11}=a_{\alpha}\left(4 P+2 x_{2} \frac{\partial P}{\partial x_{2}}\right) \\
\widetilde{\sigma}_{12}=-2 a_{\alpha} x_{2} \frac{\partial P}{\partial x_{1}} \\
\widetilde{\sigma}_{22}=-2 a_{\alpha} x_{2} \frac{\partial P}{\partial x_{2}}
\end{array} .\right.
$$

Since $P$ est harmonic on $R$, we also have

$$
\left\{\begin{array}{l}
\frac{\partial \widetilde{\sigma}_{11}}{\partial x_{1}}+\frac{\partial \widetilde{\sigma}_{12}}{\partial x_{2}}=2 a_{\alpha} \frac{\partial P}{\partial x_{1}}=0 \\
\frac{\partial \widetilde{\sigma}_{12}}{\partial x_{1}}+\frac{\partial \widetilde{\sigma}_{22}}{\partial x_{2}}=-2 a_{\alpha} \frac{\partial P}{\partial x_{2}}=0
\end{array} \quad \text { in } R_{\alpha} .\right.
$$

According to the condition at infinity (22), it follows that $P \equiv 0$ and consequently $\widetilde{\sigma}_{11}=\widetilde{\sigma}_{12}=\widetilde{\sigma}_{22}=0$, which ends the proof.

Remark 6. Here the angular tensors $\boldsymbol{\Sigma}$ of superscripts I and II correspond to tractions across the interface at $\theta=0$ of tensile (mode I) and in-plane shear (mode II).

Remark 7. The displacements $u_{1}$ and $u_{2}$ associated to $\sigma$ through (25) are determined by (see RICE [15])

$$
2 \mu_{\alpha}\left(u_{1}+i u_{2}\right)=\kappa_{\alpha} \Phi_{\alpha}+(\bar{z}-z) \frac{\overline{\mathrm{d} \Phi_{\alpha}}}{\mathrm{d} z}-\overline{\Omega_{\alpha}},
$$


with

$$
\left\{\begin{array}{c}
\frac{\mathrm{d} \Phi_{\alpha}}{\mathrm{d} z} \equiv \exp \left(\pi_{\alpha} \varepsilon\right) z^{-\frac{1}{2}-i \varepsilon} f \\
\frac{\mathrm{d} \Omega_{\alpha}}{\mathrm{d} z} \equiv \exp \left(-\pi_{\alpha} \varepsilon\right) z^{-\frac{1}{2}+i \varepsilon} \bar{f}
\end{array}, \quad f=\frac{\bar{K}}{2 \sqrt{2 \pi} \cosh (\pi \varepsilon)} .\right.
$$

Thus the discontinuity on $\Gamma_{-}$is such that

$$
\left(u_{2}+i u_{1}\right)_{\mid \theta=\pi}-\left(u_{2}+i u_{1}\right)_{\mid \theta=-\pi}=\frac{c_{1}+c_{2}}{2 \sqrt{2 \pi}(1+2 i \varepsilon) \cosh (\pi \varepsilon)} K r^{i \varepsilon} r^{\frac{1}{2}}, c_{\alpha} \equiv \frac{\kappa_{\alpha}+1}{\mu_{\alpha}} .
$$

\section{Yield Phase Transformation Surface Equations}

For the sequel, we make precise the properties we need of the loading function $\mathcal{G}$. We also give an equivalent formulation of Problem (4) and deduce an equivalent formulation of Problem (15)-(16) in order to characterize the local phase angle $\widehat{\psi}$.

Consider the yield phase transformation surface equation (4) where $\mathcal{G}$ is a convex function defined by

$$
\mathcal{G}(\boldsymbol{\sigma}) \equiv\left\{\begin{array}{cc}
\overline{\boldsymbol{\sigma}} \times g\left(y_{\boldsymbol{\sigma}}\right) & \forall \overline{\boldsymbol{\sigma}} \neq 0 \\
0 & \overline{\boldsymbol{\sigma}}=0
\end{array}, \quad g \in \boldsymbol{C}_{+}([-1,1]) .\right.
$$

In (35), $\boldsymbol{C}_{+}([-1,1])$ denotes, the set of continuous and strictly positive functions on $[-1,1]$. The Huber-Von Mises equivalent stress $\bar{\sigma}$ is given by the classical definition

$$
\bar{\sigma} \equiv \sqrt{\frac{3}{2}}|\operatorname{dev}(\sigma)|
$$

and some reduced third deviatoric stress invariant

$$
y_{\sigma} \equiv \frac{27}{2} \frac{\operatorname{det}(\operatorname{dev}(\sigma))}{\bar{\sigma}^{3}} .
$$

We note that $y_{\sigma} \in[-1,1]$ (see Remark 9).

Here $|\mathbf{a}|$ is the Frobenius norm of tensor a defined by

$$
|\mathbf{a}| \equiv \sqrt{\operatorname{tr}\left(\mathbf{a}^{T} \mathbf{a}\right)}
$$

$\operatorname{tr}($.$) denotes the trace operator and the stress deviatoric tensor is$

$$
\operatorname{dev}(\boldsymbol{\sigma}) \equiv \boldsymbol{\sigma}-\frac{1}{3} \operatorname{tr}(\boldsymbol{\sigma}) \mathbf{1},
$$

$\mathbf{1}$ being the identity tensor.

Remark 8. Function $g$ is introduced in order to take into account the asymmetry between tension and compression in SMAs and other alloys whose behavior is independent of pressure, as is the case in plasticity (see RANIECKI and LEXCELLENT [16], Bouvet et al. [17] and their references).

If one takes $g \equiv 1$, the loading function $\mathcal{G}$ is a Huber-Von Mises function which does not take into account the asymmetry between tension and compression as presented by PANOSKALtSIS et al. [5] or RANIECKI and LEXCELLENT [16].

We note that $g \equiv 1$ in FREED et al. [4], while here we take a more general $g$ (see $(35))$. 
Remark 9. We note that

$$
y_{\sigma} \in[-1,1] \forall \bar{\sigma} \neq 0 .
$$

Indeed, for all traceless symmetric matrix $\mathbf{s} \equiv \operatorname{dev}(\boldsymbol{\sigma}) \neq \mathbf{0}$ with eigenvalues $\left(\eta_{1}, \eta_{2}, \eta_{3}\right)$, we have

$$
\left|y_{\sigma}\right|^{2}=\frac{27}{4} \frac{\eta_{1}^{2} \eta_{2}^{2}\left(\eta_{1}+\eta_{2}\right)^{2}}{\left(\eta_{1} \eta_{2}+\eta_{1}^{2}+\eta_{2}^{2}\right)^{3}} \leqq 1
$$

because

$$
\left(\eta_{1} \eta_{2}+\eta_{1}^{2}+\eta_{2}^{2}\right)^{3}-\frac{27}{4} \eta_{1}^{2} \eta_{2}^{2}\left(\eta_{1}+\eta_{2}\right)^{2}=\frac{1}{4}\left(\eta_{2}-\eta_{1}\right)^{2}\left(\eta_{1}+2 \eta_{2}\right)^{2}\left(2 \eta_{1}+\eta_{2}\right)^{2} \geqq 0 .
$$

We note also that the map $\sigma \rightarrow y_{\sigma} \in[-1,1]$ is onto because for any $y \in[-1,1]$, the matrix

$$
\boldsymbol{\sigma}=\left(\begin{array}{ccc}
\eta_{1}(y) & 0 & 0 \\
0 & \eta_{2}(y) & 0 \\
0 & 0 & \eta_{3}(y)
\end{array}\right)
$$

with

$$
\eta_{k}(y)=\cos \left(\frac{\arccos (y)+2 k \pi}{3}\right), \quad k=1,2,3
$$

is such that $y_{\sigma}=y$ (a consequence of $\eta_{1}+\eta_{2}+\eta_{3}=0, \operatorname{det}(\mathbf{s})=\frac{1}{4} y$ and $\bar{\sigma}=\frac{3}{2}$ ).

Remark 10. Let $\mathbb{M}_{3}$ be the vector space of real square matrices of order 3 and let $\mathbb{S}_{3}$ the subspace of symmetric matrices. Clearly, the function

$$
\mathcal{G}: \mathbb{S}_{3} \rightarrow \mathbb{R}
$$

defined by (35), has the following properties:

$$
\mathcal{G}(\sigma)=\mathcal{G}(\operatorname{dev}(\sigma)) \forall \sigma \in \mathbb{S}_{3},
$$

is positively homogeneous of degree 1 , that is,

$$
\mathcal{G}(t \sigma)=t \mathcal{G}(\sigma) \forall \sigma \in \mathbb{S}_{3}, \quad \forall t \geqq 0,
$$

and is invariant under orthogonal transformation, that is,

$$
M \in \mathbb{M}_{3}: M M^{T}=\mathbf{1} \Rightarrow \mathcal{G}(\boldsymbol{\sigma})=\mathcal{G}\left(M^{T} \boldsymbol{\sigma} M\right) \forall \boldsymbol{\sigma} \in \mathbb{S}_{3}
$$

Let us point out that Properties (37)-(39) are verified by classical loading functions such as those of Huber-Von Mises and Tresca (see [18]). Note that the reciprocal implication holds true for convex functions as we show in the next Proposition. 
Proposition 1. Any not identically zero convex function $\mathcal{G}: \mathbb{S}_{3} \rightarrow \mathbb{R}$, satisfying Properties (37)-(39) can (necessarily) be written in the form (35).

Proof. Indeed, the case $\bar{\sigma}=0$ is trivial. Let us check (35) for $\sigma \in \mathbb{S}_{3}$, such that $\bar{\sigma} \neq 0$. Let $\mathbf{n}_{\sigma} \equiv \frac{3}{2} \frac{1}{\bar{\sigma}} \operatorname{dev}(\boldsymbol{\sigma})$. It is easy to see that

$$
\mathcal{G}(\boldsymbol{\sigma})=\mathcal{G}(\operatorname{dev}(\boldsymbol{\sigma}))=\frac{2}{3} \overline{\boldsymbol{\sigma}} \mathcal{G}\left(\mathbf{n}_{\boldsymbol{\sigma}}\right)=\frac{2}{3} \overline{\boldsymbol{\sigma}} \mathcal{G}\left(\begin{array}{ccc}
\eta_{1}\left(y_{\boldsymbol{\sigma}}\right) & 0 & 0 \\
0 & \eta_{2}\left(y_{\boldsymbol{\sigma}}\right) & 0 \\
0 & 0 & \eta_{3}\left(y_{\boldsymbol{\sigma}}\right)
\end{array}\right)
$$

where the functions $\eta_{k}$ are given by (36) (see (73) for the eigenvalues of $\mathbf{n}_{\sigma}$ ). Thus (see Remark 9))

$$
g: y \in[-1,1] \rightarrow g(y)=\frac{2}{3} \mathcal{G}\left(\begin{array}{ccc}
\eta_{1}(y) & 0 & 0 \\
0 & \eta_{2}(y) & 0 \\
0 & 0 & \eta_{3}(y)
\end{array}\right)
$$

The continuity of $g$ follows immediately from that of $\mathcal{G}$ and of $\eta_{k}$. The strict positivity of $g$ is a direct consequence of the double inequality (68) in Lemma 1 (note that the proof of (68) does not use the positivity of $g$ ). It suffices to see that $g(0)>0$ (the case $g(0)=0$ being excluded because $\mathcal{G}$ not identically zero by assumption).

Remark 11. Note that a positively homogeneous (of degree 1) map

$$
\mathcal{G}: \mathbb{S}_{3} \rightarrow[0,+\infty[
$$

is convex if and only if the domain

$$
\mathcal{E} \equiv\left\{\sigma \in \mathbb{S}_{3} \mid \mathcal{G}(\sigma) \leqq \sigma_{c}\right\},\left(\sigma_{c}>0 \text { being fixed }\right),
$$

is convex. In practice, $\mathcal{E}$ represents the elasticity domain of the material and $\sigma_{c}$ is its yield value. Experiments show that the domain $\mathcal{E}$ is, in general, convex. This motivates the convexity assumption on the function $\mathcal{G}$.

\subsection{Surface Radius Expression}

Proposition 2. We assume (14) with constants $\sigma_{c}$ and $K$ given, respectively, by (5) and (23). Then, for $\sigma$ and $\mathcal{G}$ defined, respectively, by (25) and (35), Problem (4) amounts to finding $r>0$ such that

$$
r=L \times \rho^{2} \times|\mathcal{G}(\Sigma(\widetilde{\psi}(r, \varepsilon), \theta, \varepsilon, \bar{v}))|^{2} \forall \theta \in[0, \pi[,
$$

where

$$
\begin{aligned}
L & \equiv \frac{1}{2 \pi}\left(\frac{|K|}{\sigma_{M}}\right)^{2}, \\
\rho(\xi) & \equiv\left|(1-\xi) \frac{\sigma_{A}}{\sigma_{M}}+\xi\right|^{-1} \geqq 1 \forall \xi \in[0,1], \\
\widetilde{\psi}(r, \varepsilon) & =\psi+\varepsilon \ln \left(\frac{r}{\widehat{L}}\right) \forall r>0,
\end{aligned}
$$


and, for all arguments $\varphi, \Sigma(\varphi, \theta, \varepsilon, \bar{v})$ is defined by

$$
\Sigma(\varphi, \theta, \varepsilon, \bar{v})=\cos (\varphi) \times \Sigma^{I}(\theta, \varepsilon, \bar{v})+\sin (\varphi) \times \Sigma^{I I}(\theta, \varepsilon, \bar{v}),
$$

where $\Sigma^{I}$ and $\Sigma^{I I}$ are given either by (26) or by (86).

Proof. This result is a direct consequence of the invariance of $\mathcal{G}$ (39) and its homogeneity (38). The stress tensor $\sigma$ can be written as (see (25))

$$
\left.\boldsymbol{\sigma}(r, \theta, \varepsilon, \bar{v})=\frac{|K|}{\sqrt{2 \pi r}} \boldsymbol{\Sigma}(\widetilde{\psi}(r, \varepsilon), \theta, \varepsilon, \bar{v}) \forall \theta \in\right]-\pi, \pi[.
$$

One can write the yield surface equation (4) as $(\theta \in[0, \pi[)$

$$
\frac{|K|}{\sqrt{2 \pi r}} \mathcal{G}(\Sigma(\widetilde{\psi}(r, \varepsilon), \theta, \varepsilon, \bar{v}))=\sigma_{c}=\sigma_{M} \rho^{-1} .
$$

The remainder is clear.

\subsection{Final Expression of the Local Phase Angle}

Equation (16) is a particular case of (4), where $\theta=0$.

Thus, the $\widehat{\psi}$-problem (15)-(16) amounts finally to looking for $\widehat{\psi}=\widetilde{\psi}_{\mid \Gamma_{+}}$or equivalently to solving (41) with $\theta=0$. The $\widehat{\psi}$ value is obtained by

$$
\left\{\begin{array}{l}
\widehat{\psi}=\psi+\varepsilon \ln \left(\frac{r}{\widehat{L}}\right) \\
r=L \times \rho^{2} \times|\mathcal{G}(\widehat{\boldsymbol{\Sigma}}(\widehat{\psi}))|^{2}
\end{array},\right.
$$

where, for all arguments $\varphi, \widehat{\boldsymbol{\Sigma}}(\varphi)$ is defined by (see (45))

$$
\widehat{\mathbf{\Sigma}}(\varphi) \equiv \mathbf{\Sigma}(\varphi, \theta=0, \varepsilon, \bar{v}) .
$$

Using the fact that $\varepsilon$ depends on $\beta$ (see (6)), the tensor $\widehat{\boldsymbol{\Sigma}}$ (.) can be written in terms of fixed $\beta$ and $\bar{v}$ as

$$
\widehat{\boldsymbol{\Sigma}}(\varphi)=\cos (\varphi) \times \mathcal{C}(\beta, \bar{v})+\sin (\varphi) \times \mathcal{S},
$$

where $\mathcal{C} \equiv \Sigma^{I}(0, \varepsilon, \bar{v})$ and $\mathcal{S} \equiv \Sigma^{I I}(0, \varepsilon, \bar{v})($ see $(26))$. More explicitly

$$
\mathcal{C}(\beta, \bar{v})=\left(\begin{array}{lll}
2 \beta+1 & 0 & 0 \\
0 & 1 & 0 \\
0 & 0 & 2 \bar{v}
\end{array}\right) \text { and } \mathcal{S}=\left(\begin{array}{lll}
0 & 1 & 0 \\
1 & 0 & 0 \\
0 & 0 & 0
\end{array}\right) \text {. }
$$

With the notations above we have:

Corollary 1. Under the same assumptions as in Proposition 2, let $\widehat{\psi}$ be a solution to (15)-(16). Then, besides the trivial case of $\varepsilon=0$ where $\widehat{\psi}=\psi$, the value of $\widehat{\psi}$ is a solution of the nonlinear equation

$$
\widehat{\psi}=\mathcal{O}(\widehat{\psi})
$$

where

$$
\mathcal{O}: \varphi \in \mathbb{R} \rightarrow \mathcal{O}(\varphi) \equiv \psi+\varepsilon \ln \left(\frac{L}{\widehat{L}} \times \rho^{2}|\mathcal{G}(\widehat{\boldsymbol{\Sigma}}(\varphi))|^{2}\right)
$$




\section{Main Results}

Our main goal is to study the fixed point problem (51) where the parameters $\psi, \varepsilon, L, \widehat{L}, \rho, \beta$ and $\bar{v}$ are fixed. We start with :

Theorem 2. Let $\mathcal{G}$, given by (35), be convex and let $\widehat{\mathbf{\Sigma}}$ be given by (49). The set of solutions to Problem (51) is not empty and any solution $\widehat{\psi}$ satisfies the estimate

$$
u \leqq \psi-\widehat{\psi} \leqq v,
$$

where

$$
u \equiv\left\{\begin{array}{ll}
-\omega^{(g)} & \text { if } \varepsilon \geqq 0 \\
\left(\omega^{(g)}+\varpi\right) & \text { if } \varepsilon \leqq 0
\end{array}, \quad v \equiv \begin{cases}-\left(\omega^{(g)}+\varpi\right) & \text { if } \varepsilon \geqq 0 \\
\omega^{(g)} & \text { if } \varepsilon \leqq 0\end{cases}\right.
$$

and

$$
\begin{aligned}
\omega^{(g)} & \equiv|\varepsilon| \ln \left(4 \frac{L}{L} \times \rho^{2} \times|g(0)|^{2}\right), \quad \varpi \equiv 2|\varepsilon| \ln \left(\frac{1}{2} \delta\right), \\
\delta & \equiv \sqrt{\beta^{2}+\frac{1}{3}(\beta+(1-2 \bar{\nu}))^{2}} .
\end{aligned}
$$

Moreover, if

$$
k_{\varepsilon} \equiv \frac{4|\varepsilon|}{\delta}<1
$$

is satisfied, then the solution $\widehat{\psi}$ is unique and can be computed by the iterative method

$$
\widehat{\psi}_{(0)}=\psi, \widehat{\psi}_{(n+1)}=\mathcal{O}\left(\widehat{\psi}_{(n)}\right) \forall n \in \mathbb{N}
$$

and the following estimate holds

$$
\left|\widehat{\psi}-\widehat{\psi}_{(n+1)}\right| \leqq 2|\varepsilon| k_{\varepsilon}^{n} \ln \frac{2}{\delta} .
$$

We complement Theorem 2 by

\section{Proposition 3. Under}

$$
e \equiv \frac{E_{2}}{E_{1}} \leqq 1 \quad \text { and } \quad v_{1}=v_{2}=v
$$

we have

$$
k_{\varepsilon}<\frac{8}{3 \pi}
$$

and then (57) is satisfied.

The proof of (61) is given in Appendix C.

We now give a comparison result which follows easily from Theorem 2. 
Corollary 2. Let $\widehat{\psi}^{\left(g_{1}\right)}$ and $\widehat{\psi}^{\left(g_{2}\right)}$ be respective solutions of (51) corresponding to $g=g_{1}$ and to $g=g_{2}$. Then

$$
2 \varepsilon \ln \left(\frac{1}{2} \delta \frac{g_{1}(0)}{g_{2}(0)}\right) \leqq \widehat{\psi}^{\left(g_{1}\right)}-\widehat{\psi}^{\left(g_{2}\right)} \leqq 2 \varepsilon \ln \left(\frac{2}{\delta} \frac{g_{1}(0)}{g_{2}(0)}\right) \forall \varepsilon \geqq 0
$$

and

$$
2 \varepsilon \ln \left(\frac{2}{\delta} \frac{g_{1}(0)}{g_{2}(0)}\right) \leqq \widehat{\psi}^{\left(g_{1}\right)}-\widehat{\psi}^{\left(g_{2}\right)} \leqq 2 \varepsilon \ln \left(\frac{1}{2} \delta \frac{g_{1}(0)}{g_{2}(0)}\right) \forall \varepsilon \leqq 0 .
$$

Proof. The above result is a corollary of (53). In fact, one has:

$$
u_{g_{i}} \leqq \psi-\widehat{\psi}^{\left(g_{i}\right)} \leqq v_{g_{i}} \text { for } i=1,2 .
$$

By substraction, one gets

$$
u_{g_{2}}-v_{g_{1}} \leqq \widehat{\psi}^{\left(g_{1}\right)}-\widehat{\psi}^{\left(g_{2}\right)} \leqq v_{g_{2}}-u_{g_{1}},
$$

with

$$
u_{g_{2}}-v_{g_{1}}=\left\{\begin{array}{ll}
\varpi+\omega^{\left(g_{1}\right)}-\omega^{\left(g_{2}\right)} & \text { if } \varepsilon \geqq 0 \\
\varpi-\left(\omega^{\left(g_{1}\right)}-\omega^{\left(g_{2}\right)}\right) & \text { if } \varepsilon \leqq 0
\end{array}=\left\{\begin{array}{l}
2 \varepsilon \ln \left(\frac{1}{2} \delta \frac{g_{1}(0)}{g_{2}(0)}\right) \text { if } \varepsilon \geqq 0 \\
2 \varepsilon \ln \left(\frac{2}{\delta} \frac{g_{1}(0)}{g_{2}(0)}\right) \text { if } \varepsilon \leqq 0
\end{array}\right.\right.
$$

and

$v_{g_{2}}-u_{g_{1}}=\left\{\begin{array}{ll}\left(\omega^{\left(g_{1}\right)}-\omega^{\left(g_{2}\right)}\right)-\varpi & \text { if } \varepsilon \geqq 0 \\ -\left(\omega^{\left(g_{1}\right)}-\omega^{\left(g_{2}\right)}\right)-\varpi & \text { if } \varepsilon \leqq 0\end{array}=\left\{\begin{array}{l}2 \varepsilon \ln \left(\frac{2}{\delta} \frac{g_{1}(0)}{g_{2}(0)}\right) \text { if } \varepsilon \geqq 0 \\ 2 \varepsilon \ln \left(\frac{1}{2} \delta \frac{g_{1}(0)}{g_{2}(0)}\right) \text { if } \varepsilon \leqq 0\end{array}\right.\right.$.

We finally have (62) and (63)

\section{Proof of Theorem 2}

The proof of Theorem 2 uses the following proposition.

Proposition 4. Let $\mathcal{G}$, given by (35), be convex and let $\widehat{\boldsymbol{\Sigma}}$ be given by (49). Then

$$
0<g(0) \delta \leqq \mathcal{G}(\widehat{\boldsymbol{\Sigma}}(\varphi) \leqq 2 g(0) \forall \varphi \in \mathbb{R}
$$

and

$$
2|\varepsilon|\left|\ln \left(\frac{\mathcal{G}\left(\widehat{\boldsymbol{\Sigma}}\left(\varphi_{2}\right)\right)}{\mathcal{G}\left(\widehat{\boldsymbol{\Sigma}}\left(\varphi_{1}\right)\right)}\right)\right| \leqq k_{\varepsilon}\left|\varphi_{2}-\varphi_{1}\right| \quad \forall \varphi_{1}, \varphi_{2} \in \mathbb{R}
$$

(see (56) and (57)).

This proposition follows from the following general estimates of independent interest; in particular, we show how the convexity of $\mathcal{G}$ implies suitable bounds on $g$. 
Lemma 1. We assume that $\mathcal{G}$ given by (35) is convex. Then

$$
\frac{2}{3} \sqrt{3} g(0) g_{\min }(y) \leqq g(y) \leqq \frac{2}{3} \sqrt{3} g(0) g_{\max }(y) \forall y \in[-1,1]
$$

with

$$
g_{\min }(y) \equiv \sin \left(\frac{1}{6} \pi+\frac{1}{3} \arccos |y|\right), \quad g_{\max }(y) \equiv \cos \left(\frac{1}{3} \arccos |y|\right) .
$$

In particular

$$
\frac{1}{3} \sqrt{3} g(0) \bar{\sigma} \leqq \mathcal{G}(\sigma) \leqq \frac{2}{3} \sqrt{3} g(0) \bar{\sigma}
$$

\subsection{Proof of Lemma 1}

Proof (66). Let us show first that for all traceless symmetric matrices $\mathbf{S} \equiv \operatorname{dev}(\boldsymbol{\sigma}) \neq$ 0 with eigenvalues

$$
\lambda_{1}(\mathbf{s}) \leqq \lambda_{2}(\mathbf{s}) \leqq \lambda_{3}(\mathbf{s}),
$$

we have

$$
\sqrt{3} g(0) \mathcal{G}_{\min }(\mathbf{s}) \leqq \mathcal{G}(\sigma) \leqq \sqrt{3} g(0) \mathcal{G}_{\max }(\mathbf{s})
$$

where

$$
\mathcal{G}_{\min }(\mathbf{s}) \equiv \min \left\{\left|\lambda_{1}(\mathbf{s})\right|, \lambda_{3}(\mathbf{s})\right\} ; \mathcal{G}_{\max }(\mathbf{s}) \equiv \max \left\{\left|\lambda_{1}(\mathbf{s})\right|, \lambda_{3}(\mathbf{s})\right\}
$$

We use repeatedly the convexity of $\mathcal{G}$ and the homogeneity property. We have

$$
\mathcal{G}(\boldsymbol{\sigma})=\mathcal{G}(\mathbf{s})=\mathcal{G}(\Lambda), \text { where } \Lambda \equiv\left(\begin{array}{lll}
\lambda_{1} & 0 & 0 \\
0 & \lambda_{2} & 0 \\
0 & 0 & \lambda_{3}
\end{array}\right) \text {, with } \lambda_{1}+\lambda_{2}+\lambda_{3}=0
$$

Thus by rewriting $\Lambda$ as

$$
\Lambda=\left|\lambda_{1}\right| \Lambda_{1}+\left|\lambda_{2}\right| \Lambda_{2}
$$

with

$$
\begin{aligned}
& \Lambda_{1} \equiv \operatorname{sign}\left(\lambda_{1}\right)\left(\begin{array}{lll}
1 & 0 & 0 \\
0 & 0 & 0 \\
0 & 0 & -1
\end{array}\right), \Lambda_{2} \equiv \operatorname{sign}\left(\lambda_{2}\right)\left(\begin{array}{lll}
0 & 0 & 0 \\
0 & 1 & 0 \\
0 & 0 & -1
\end{array}\right) ; \\
& \operatorname{sign}(\lambda) \equiv \begin{cases}-1 & \lambda<0 \\
0 & \lambda=0 \\
1 & \lambda>0\end{cases}
\end{aligned}
$$

we obtain (by convexity and homogeneity of $\mathcal{G}$ ), on the one hand

$$
\mathcal{G}(\Lambda) \leqq \mathcal{G}\left(\left|\lambda_{1}\right| \Lambda_{1}\right)+\mathcal{G}\left(\left|\lambda_{2}\right| \Lambda_{2}\right)=\left|\lambda_{1}\right| \mathcal{G}\left(\Lambda_{1}\right)+\left|\lambda_{2}\right| \mathcal{G}\left(\Lambda_{2}\right),
$$


and on the other hand

$$
\begin{aligned}
\left|\lambda_{1}\right| \mathcal{G}\left(\Lambda_{1}\right) & =\mathcal{G}\left(\left|\lambda_{1}\right| \Lambda_{1}\right)=\mathcal{G}\left(\Lambda-\left|\lambda_{2}\right| \Lambda_{2}\right) \leqq \mathcal{G}(\Lambda)+\mathcal{G}\left(-\left|\lambda_{2}\right| \Lambda_{2}\right) \\
& =\mathcal{G}(\Lambda)+\left|\lambda_{2}\right| \mathcal{G}\left(-\Lambda_{2}\right)
\end{aligned}
$$

that is,

$$
\left|\lambda_{1}\right| \mathcal{G}\left(\Lambda_{1}\right)-\left|\lambda_{2}\right| \mathcal{G}\left(-\Lambda_{2}\right) \leqq \mathcal{G}(\Lambda)
$$

or by changing the suffix

$$
\left|\lambda_{2}\right| \mathcal{G}\left(\Lambda_{2}\right)-\left|\lambda_{1}\right| \mathcal{G}\left(-\Lambda_{1}\right) \leqq \mathcal{G}(\Lambda) .
$$

Now as

$$
\left.\begin{array}{l}
\overline{ \pm \Lambda}_{i}=\sqrt{3} \\
\operatorname{det} \pm \Lambda_{i}=0 \Rightarrow y_{ \pm \Lambda_{i}}=0
\end{array}\right\} \Rightarrow \mathcal{G}\left( \pm \Lambda_{i}\right)=\sqrt{3} g(0)
$$

of (70)-(71), we end up with

$$
\sqrt{3} g(0)|| \lambda_{1}|-| \lambda_{2}|| \leqq \mathcal{G}(\Lambda) \leqq \sqrt{3} g(0)\left(\left|\lambda_{1}\right|+\left|\lambda_{2}\right|\right) .
$$

In fact, for all decompositions of type $\Lambda=\left|\lambda_{i}\right| \Lambda_{i}+\left|\lambda_{j}\right| \Lambda_{j} \forall 1 \leqq i \neq j \leqq 3$, one similarly obtains

$$
\sqrt{3} g(0)|| \lambda_{i}|-| \lambda_{j}|| \leqq \mathcal{G}(\Lambda) \leqq \sqrt{3} g(0)\left(\left|\lambda_{i}\right|+\left|\lambda_{j}\right|\right) \forall 1 \leqq i \neq j \leqq 3,
$$

hence

$$
\sqrt{3} g(0) \mathcal{G}_{\min }(\mathbf{s}) \leqq \mathcal{G}(\Lambda) \leqq \sqrt{3} g(0) \mathcal{G}_{\max }(\mathbf{s})
$$

for

$$
\left\{\begin{array}{l}
\mathcal{G}_{\max }(\mathbf{s}) \equiv \inf _{1 \leqq i \neq j \leqq 3}\left(\left|\lambda_{i}\right|+\left|\lambda_{j}\right|\right) \\
\mathcal{G}_{\min }(\mathbf{s}) \equiv \max _{1 \leqq i \neq j \leqq 3}\left(|| \lambda_{i}|-| \lambda_{j}||\right)
\end{array} .\right.
$$

Finally, it suffices to note that for $\lambda_{2} \geqq 0$, one has

$$
\left.\begin{array}{c}
\left|\lambda_{1}\right|=-\lambda_{1}=\lambda_{2}+\lambda_{3}=\left|\lambda_{2}\right|+\left|\lambda_{3}\right| \\
0 \leqq \lambda_{2} \leqq \lambda_{3}
\end{array}\right\} \Rightarrow\left|\lambda_{2}\right| \leqq\left|\lambda_{3}\right| \leqq\left|\lambda_{1}\right|,
$$

therefore

$$
\left\{\begin{array}{l}
\mathcal{G}_{\max }(\mathbf{s})=\left|\lambda_{2}\right|+\left|\lambda_{3}\right|=\lambda_{2}+\lambda_{3}=-\lambda_{1}=\left|\lambda_{1}\right|=\max \left\{\left|\lambda_{1}\right|,\left|\lambda_{3}\right|\right\} \\
\mathcal{G}_{\min }(\mathbf{s})=\left|\lambda_{1}\right|-\left|\lambda_{2}\right|=-\lambda_{1}-\lambda_{2}=\lambda_{3}=\left|\lambda_{3}\right|=\min \left\{\left|\lambda_{1}\right|,\left|\lambda_{3}\right|\right\}
\end{array},\right.
$$

and for $\lambda_{2} \leqq 0$, one has

$$
\left.\begin{array}{c}
\left|\lambda_{1}\right|=-\lambda_{1}=\lambda_{2}+\lambda_{3}=-\left|\lambda_{2}\right|+\left|\lambda_{3}\right| \\
\lambda_{1} \leqq \lambda_{2} \leqq 0
\end{array}\right\} \Rightarrow\left|\lambda_{2}\right| \leqq\left|\lambda_{1}\right| \leqq\left|\lambda_{3}\right|,
$$


which implies

$$
\left\{\begin{array}{l}
\mathcal{G}_{\max }(\mathbf{s})=\left|\lambda_{1}\right|+\left|\lambda_{2}\right|=-\lambda_{1}-\lambda_{2}=\left|\lambda_{3}\right|=\max \left\{\left|\lambda_{1}\right|,\left|\lambda_{3}\right|\right\} \\
\mathcal{G}_{\min }(\mathbf{s})=\left|\lambda_{3}\right|-\left|\lambda_{2}\right|=\lambda_{3}+\lambda_{2}=-\lambda_{1}=\left|\lambda_{1}\right|=\min \left\{\left|\lambda_{1}\right|,\left|\lambda_{3}\right|\right\}
\end{array} .\right.
$$

This ends the proof of (68).

Thus

$$
\sqrt{3} g(0) \mathcal{G}_{\min }\left(\mathbf{n}_{\sigma}\right) \leqq \mathcal{G}\left(\mathbf{n}_{\sigma}\right) \equiv \underbrace{\overline{\mathbf{n}}_{\sigma}}_{=\frac{3}{2}} g(\underbrace{y_{\mathbf{n}_{\sigma}}}_{=y_{\sigma}}) \leqq \sqrt{3} g(0) \mathcal{G}_{\max }\left(\mathbf{n}_{\sigma}\right)
$$

with

$$
\mathbf{n}_{\sigma}=\frac{3}{2} \frac{1}{\bar{\sigma}} \mathbf{S}_{\sigma}
$$

That is,

$$
\frac{2}{3} \sqrt{3} g(0) \mathcal{G}_{\min }\left(\mathbf{n}_{\sigma}\right) \leqq g\left(y_{\sigma}\right) \leqq \frac{2}{3} \sqrt{3} g(0) \mathcal{G}_{\max }\left(\mathbf{n}_{\sigma}\right) .
$$

To prove (66) it suffices to compute the eigenvalues of $\mathbf{n}_{\sigma}$. The characteristic polynomial is given by

$$
\begin{aligned}
\operatorname{det}\left(\mathbf{n}_{\sigma}-\eta \mathbf{1}\right) & =-\eta^{3}+\underbrace{I_{1}}_{\equiv \operatorname{tr}\left(\mathbf{n}_{\sigma}\right)=0} \eta^{2}-\underbrace{I_{2}}_{\equiv \frac{1}{2}\left(\operatorname{tr}\left(\mathbf{n}_{\sigma}\right)^{2}-\operatorname{tr}\left(\mathbf{n}_{\sigma}^{2}\right)\right)=-\frac{3}{4}} \eta \underbrace{I_{3}}_{\operatorname{det}\left(\mathbf{n}_{\sigma}\right)=\frac{1}{4} y_{\sigma}} \\
& =-\eta^{3}+\frac{3}{4} \eta+\frac{1}{4} y_{\sigma},
\end{aligned}
$$

and a simple calculus gives the eigenvalues of $\mathbf{n}_{\sigma}$ in the nondecreasing order

$$
\lambda_{k}\left(\mathbf{n}_{\sigma}\right)=\eta_{k}\left(y_{\sigma}\right) \equiv \cos \left(\frac{\arccos \left(y_{\sigma}\right)+2 k \pi}{3}\right), \quad k=1,2,3 .
$$

Indeed, using the identity

$$
-\cos ^{3}(x)+\frac{3}{4} \cos (x)+\frac{1}{4} \cos 3 x=0 \forall x \in \mathbb{R}
$$

with $x \equiv \frac{\arccos \left(y_{\sigma}\right)+2 k \pi}{3}$, one sees that the $\eta_{k}^{\prime}$ s are the roots of the characteristic polynomial. One the other hand, with $\varrho \equiv \cos \left(\theta_{\sigma}\right) \in\left[\frac{1}{2}, 1\right]$, where $\theta_{\sigma} \equiv \frac{1}{3} \arccos \left(y_{\sigma}\right)$, we have

$\eta_{1}\left(y_{\sigma}\right)=-\frac{1}{2} \varrho-\frac{\sqrt{3}}{2} \sqrt{1-\varrho^{2}} \leqq \eta_{2}\left(y_{\sigma}\right)=-\frac{1}{2} \varrho+\frac{\sqrt{3}}{2} \sqrt{1-\varrho^{2}} \leqq \eta_{3}\left(y_{\sigma}\right)=\varrho$.

Besides, one has

$$
-\eta_{1}\left(y_{\sigma}\right)=-\cos \left(\theta_{\sigma}+\frac{2 \pi}{3}\right)=\sin \left(\frac{1}{6} \pi+\theta_{\sigma}\right) .
$$

But as

$$
\arccos \left(-y_{\sigma}\right)=\pi-\arccos \left(y_{\sigma}\right) \Rightarrow \frac{1}{3} \arccos \left|y_{\sigma}\right|=\left\{\begin{array}{cc}
\frac{\pi}{3}-\theta_{\sigma} & \text { if } y_{\sigma} \leqq 0 \\
\theta_{\sigma} & \text { if } y_{\sigma} \geqq 0
\end{array},\right.
$$


this implies

$$
\begin{aligned}
g_{\min }\left(y_{\boldsymbol{\sigma}}\right) & \equiv \sin \left(\frac{\pi}{6}+\frac{1}{3} \arccos \left|y_{\sigma}\right|\right)=\left\{\begin{array}{cc}
\cos \theta_{\sigma} & \text { if } y_{\boldsymbol{\sigma}} \leqq 0 \\
\sin \left(\frac{\pi}{6}+\theta_{\sigma}\right) & \text { if } y_{\boldsymbol{\sigma}} \geqq 0
\end{array}\right. \\
& =\left\{\begin{array}{c}
\eta_{3}\left(y_{\boldsymbol{\sigma}}\right) \text { if } y_{\boldsymbol{\sigma}} \leqq 0 \\
-\eta_{1}\left(y_{\boldsymbol{\sigma}}\right) \text { if } y_{\boldsymbol{\sigma}} \geqq 0
\end{array}=\left\{\begin{array}{c}
\eta_{3}\left(y_{\boldsymbol{\sigma}}\right) \text { if } \eta_{2}\left(y_{\boldsymbol{\sigma}}\right) \geqq 0 \\
-\eta_{1}\left(y_{\boldsymbol{\sigma}}\right) \text { if } \eta_{2}\left(y_{\boldsymbol{\sigma}}\right) \leqq 0
\end{array}\right.\right.
\end{aligned}
$$

and

$$
\begin{aligned}
& g_{\max }\left(y_{\boldsymbol{\sigma}}\right) \equiv \cos \left(\frac{1}{3} \arccos \left|y_{\boldsymbol{\sigma}}\right|\right)=\left\{\begin{array}{cc}
\sin \left(\frac{1}{6} \pi+\theta_{\sigma}\right) & \text { if } y_{\boldsymbol{\sigma}} \leqq 0 \\
\cos \left(\theta_{\boldsymbol{\sigma}}\right) & \text { if } y_{\sigma} \geqq 0
\end{array}\right. \\
& =\left\{\begin{array}{c}
-\eta_{1}\left(y_{\boldsymbol{\sigma}}\right) \text { if } y_{\boldsymbol{\sigma}} \leqq 0 \\
\eta_{3}\left(y_{\boldsymbol{\sigma}}\right) \text { if } y_{\boldsymbol{\sigma}} \geqq 0
\end{array}=\left\{\begin{array}{c}
-\eta_{1}\left(y_{\boldsymbol{\sigma}}\right) \text { if } \eta_{2}\left(y_{\boldsymbol{\sigma}}\right) \geqq 0 \\
\eta_{3}\left(y_{\boldsymbol{\sigma}}\right) \text { if } \eta_{2}\left(y_{\boldsymbol{\sigma}}\right) \leqq 0
\end{array} .\right.\right.
\end{aligned}
$$

This leads to the announced result by a substitution in (72), that is

$$
\mathcal{G}_{\min }\left(\mathbf{n}_{\sigma}\right) \equiv\left\{\begin{array}{l}
\eta_{3}\left(y_{\sigma}\right) \text { for } \eta_{2}\left(y_{\sigma}\right) \geqq 0 \\
-\eta_{1}\left(y_{\sigma}\right) \text { for } \eta_{2}\left(y_{\sigma}\right) \leqq 0
\end{array}=g_{\min }\left(y_{\sigma}\right)\right.
$$

and

$$
\mathcal{G}_{\max }\left(\mathbf{n}_{\boldsymbol{\sigma}}\right) \equiv\left\{\begin{array}{ll}
-\eta_{1}\left(y_{\boldsymbol{\sigma}}\right) & \text { for } \eta_{2}\left(y_{\boldsymbol{\sigma}}\right) \geqq 0 \\
\eta_{3}\left(y_{\boldsymbol{\sigma}}\right) & \text { for } \eta_{2}\left(y_{\boldsymbol{\sigma}}\right) \leqq 0
\end{array}=g_{\max }\left(y_{\boldsymbol{\sigma}}\right) .\right.
$$

This ends the proof of (66) (see Remark 9).

Proof (67). This is a direct consequence of (66) (because $g_{\min } \geqq \frac{1}{2}$ and $g_{\max } \leqq 1$ ) and of the definition of $\mathcal{G}$.

Remark 12. The choice $y=0$ in (66) shows that the constants are optimal.

\subsection{Proof of Proposition 4}

Proof (64). The eigenvalues of

$$
\mathbf{S}(\varphi) \equiv \operatorname{dev}(\widehat{\boldsymbol{\Sigma}}(\varphi))
$$

are given by

$$
\left\{\begin{array}{l}
\eta_{1}=-2 \beta_{0} \cos \varphi \\
\eta_{2}=\beta_{0} \cos \varphi-\sqrt{\sin ^{2} \varphi+\beta^{2} \cos ^{2} \varphi} \\
\eta_{3}=\beta_{0} \cos \varphi+\sqrt{\sin ^{2} \varphi+\beta^{2} \cos ^{2} \varphi}
\end{array}\right.
$$

where

$$
\beta_{0} \equiv \frac{\beta+(1-2 \bar{v})}{3}
$$

The expression

$$
|\overline{\mathbf{\Sigma}(\varphi)}|^{2}=\frac{3}{2}|\mathbf{s}(\varphi)|^{2}=\frac{3}{2}\left(\left|\eta_{1}\right|^{2}+\left|\eta_{2}\right|^{2}+\left|\eta_{3}\right|^{2}\right)
$$


leads to

$$
\frac{1}{3}|\overline{\widehat{\boldsymbol{\Sigma}}(\varphi)}|^{2}=\delta^{2}+\left(1-\delta^{2}\right) \sin ^{2} \varphi,
$$

where

$$
\delta^{2} \equiv \beta^{2}+3 \beta_{0}^{2}=\beta^{2}+\frac{1}{3}(\beta+(1-2 \bar{\nu}))^{2} .
$$

Now as $|\beta|<\frac{1}{2}$ (see (11)) and $0<1-2 \bar{\nu} \leqq 1$, one has

$$
0<\delta^{2}<1 \text {. }
$$

We can then deduce

$$
0<\delta^{2} \leqq \frac{1}{3}|\widehat{\boldsymbol{\Sigma}}(\varphi)|^{2} \leqq 1 \forall \varphi \in \mathbb{R}
$$

Due to (67), one has

$$
\frac{1}{4} \delta^{2} \leqq \frac{1}{4}\left(\frac{1}{3}|\widehat{\boldsymbol{\Sigma}}(\varphi)|^{2}\right) \leqq\left|\frac{\mathcal{G}(\widehat{\boldsymbol{\Sigma}}(\varphi)}{2 g(0)}\right|^{2} \leqq \frac{1}{3}|\widehat{\widehat{\boldsymbol{\Sigma}}(\varphi)}|^{2} \leqq 1 \forall \varphi \in \mathbb{R}
$$

that is (64).

Proof (65). From (49),

$$
\begin{aligned}
\widehat{\boldsymbol{\Sigma}}\left(\varphi_{2}\right)-\widehat{\boldsymbol{\Sigma}}\left(\varphi_{1}\right) & =\left(\cos \left(\varphi_{2}\right)-\cos \left(\varphi_{1}\right)\right) \times \mathcal{C}+\left(\sin \left(\varphi_{2}\right)-\sin \left(\varphi_{1}\right)\right) \times \mathcal{S} \\
& =2 \sin \left(\frac{\varphi_{2}-\varphi_{1}}{2}\right)\left(\cos \left(\frac{\varphi_{2}+\varphi_{1}}{2}+\frac{\pi}{2}\right) \mathcal{C}+\sin \left(\frac{\varphi_{2}+\varphi_{1}}{2}+\frac{\pi}{2}\right) \mathcal{S}\right),
\end{aligned}
$$

that is,

$$
\widehat{\boldsymbol{\Sigma}}\left(\varphi_{2}\right)=\widehat{\boldsymbol{\Sigma}}\left(\varphi_{1}\right)+2 \sin \left(\frac{\varphi_{2}-\varphi_{1}}{2}\right) \widehat{\boldsymbol{\Sigma}}\left(\frac{\varphi_{2}+\varphi_{1}}{2}+\frac{\pi}{2}\right) .
$$

By using (64) and the convexity of $\mathcal{G}$ and its homogeneity, one also has

$$
\begin{aligned}
\mathcal{G}\left(\widehat{\boldsymbol{\Sigma}}\left(\varphi_{2}\right)\right) & \leqq \mathcal{G}\left(\widehat{\boldsymbol{\Sigma}}\left(\varphi_{1}\right)\right)+2 \underbrace{\mid \sin \left(\frac{\varphi_{2}-\varphi_{1}}{2} \mid\right.}_{\leqq} \underbrace{\mathcal{G}\left(\widehat{\boldsymbol{\Sigma}}\left(\frac{\varphi_{2}+\varphi_{1}}{2}+\frac{\pi}{2}\right)\right)}_{\leqq 2 g(0)} \\
& \leqq \mathcal{G}\left(\widehat{\boldsymbol{\Sigma}}\left(\varphi_{1}\right)\right)+2 g(0)\left|\varphi_{2}-\varphi_{1}\right|,
\end{aligned}
$$

that is,

$$
\frac{\mathcal{G}\left(\widehat{\boldsymbol{\Sigma}}\left(\varphi_{2}\right)\right)}{\mathcal{G}\left(\widehat{\boldsymbol{\Sigma}}\left(\varphi_{1}\right)\right)} \leqq 1+2 \underbrace{\left(\frac{g(0)}{\mathcal{G}\left(\widehat{\boldsymbol{\Sigma}}\left(\varphi_{1}\right)\right)}\right)}_{\leqq \frac{1}{\delta}}\left|\varphi_{2}-\varphi_{1}\right|
$$

Therefore

$$
\ln \frac{\mathcal{G}\left(\widehat{\boldsymbol{\Sigma}}\left(\varphi_{2}\right)\right)}{\mathcal{G}\left(\widehat{\boldsymbol{\Sigma}}\left(\varphi_{1}\right)\right)} \leqq \ln \left(1+\frac{2}{\delta}\left|\varphi_{2}-\varphi_{1}\right|\right) \leqq \frac{2}{\delta}\left|\varphi_{2}-\varphi_{1}\right|,
$$

and a permutation of indices leads to the result (65). 


\subsection{Proof of Theorem 2}

Proof. Equation (51) reads

$$
\widehat{\psi}-\psi=\left\{\begin{array}{l}
\omega^{(g)}+|\varepsilon| \ln \left(\left|\frac{\mathcal{G}(\widehat{\boldsymbol{\Sigma}}(\widehat{\psi}))}{2 g(0)}\right|^{2}\right) \quad \forall \varepsilon \geqq 0 \\
-\omega^{(g)}-|\varepsilon| \ln \left(\left|\frac{\mathcal{G}(\widehat{\boldsymbol{\Sigma}}(\widehat{\psi}))}{2 g(0)}\right|^{2}\right) \forall \varepsilon \leqq 0
\end{array} .\right.
$$

Therefore, by the change of variable

$$
\begin{aligned}
\widehat{\varphi} & \equiv f(\widehat{\psi}) \equiv\left\{\begin{array}{l}
\widehat{\psi}-\psi-\omega^{(g)} \text { if } \varepsilon \geqq 0 \\
\psi-\widehat{\psi}-\omega^{(g)} \text { if } \varepsilon \leqq 0
\end{array} \Leftrightarrow \widehat{\psi} \equiv f^{-1}(\widehat{\varphi})\right. \\
& \equiv\left\{\begin{array}{l}
\widehat{\varphi}+\psi+\omega^{(g)} \text { if } \varepsilon \geqq 0 \\
\psi-\widehat{\varphi}-\omega^{(g)} \text { if } \varepsilon \leqq 0
\end{array},\right.
\end{aligned}
$$

the equation (51) becomes

$$
\widehat{\varphi}=\mathcal{L}(\widehat{\varphi})
$$

where

$$
\mathcal{L}(\varphi)=|\varepsilon| \ln \left(\left|\frac{\mathcal{G}\left(\widehat{\Sigma}\left(f^{-1}(\varphi)\right)\right)}{2 g(0)}\right|^{2}\right) \forall \varphi \in \mathbb{R} .
$$

Thanks to the estimate (64), that is,

$$
\frac{1}{4} \delta^{2} \leqq\left|\frac{\mathcal{G}(\widehat{\Sigma}(\varphi))}{2 g(0)}\right|^{2} \leqq 1 \forall \varphi \in \mathbb{R},
$$

one gets

$$
\varpi \leqq \mathcal{L}(\varphi) \leqq 0 \forall \varphi \in \mathbb{R}
$$

Hence the function

$$
\varphi \in[\varpi, 0] \rightarrow z(\varphi) \equiv \varphi-\mathcal{L}(\varphi),
$$

which is continuous with respect to $\varphi$, changes sign on $[\varpi, 0]$, that is

$$
z(\varpi)=\varpi-\mathcal{L}(\varpi) \leqq 0 \text { and } z(0)=0-\mathcal{L}(0) \geqq 0 .
$$

Therefore its graph necessarily passes through the origin. There exists, then, at least $\widehat{\varphi} \in[\varpi, 0]$, such that

$$
\widehat{\varphi}=\mathcal{L}(\widehat{\varphi}) .
$$

The solution is finally deduced by

$$
\widehat{\psi} \equiv f^{-1}(\widehat{\varphi}) \text { with } \widehat{\varphi} \in[\varpi, 0],
$$

which implies (53).

The uniqueness of $\widehat{\varphi}$ easily results from (65) and hypothesis (57). In fact, for $\widehat{\varphi}_{i}=\mathcal{L}\left(\widehat{\varphi}_{i}\right), i=1,2$, one has 


$$
\begin{aligned}
\left|\widehat{\varphi}_{2}-\widehat{\varphi}_{1}\right| & =2|\varepsilon| \mid \ln \left(\mathcal{G}\left(\widehat{\boldsymbol{\Sigma}}\left(f^{-1}\left(\widehat{\varphi}_{2}\right)\right)\right)-\ln \left(\mathcal{G}\left(\widehat{\boldsymbol{\Sigma}}\left(f^{-1}\left(\widehat{\varphi}_{1}\right)\right)\right) \mid\right.\right. \\
& \leqq k_{\varepsilon}\left|f^{-1}\left(\widehat{\varphi}_{2}\right)-f^{-1}\left(\widehat{\varphi}_{1}\right)\right|=k_{\varepsilon}\left|\widehat{\varphi}_{2}-\widehat{\varphi}_{1}\right| .
\end{aligned}
$$

Thus $\widehat{\varphi}_{2}=\widehat{\varphi}_{1}$.

Finally, the estimate (59) is a direct consequence of (65) and of (53). Indeed, for all $n \in \mathbb{N}$, one has

$$
\begin{aligned}
\left|\widehat{\psi}-\widehat{\psi}_{(n+1)}\right| & =\left|\mathcal{O}_{g}(\widehat{\psi})-\mathcal{O}_{g}\left(\widehat{\psi}_{(n)}\right)\right|=2|\varepsilon|\left|\ln \left(\frac{\mathcal{G}(\widehat{\boldsymbol{\Sigma}}(\widehat{\psi}))}{\mathcal{G}\left(\widehat{\boldsymbol{\Sigma}}\left(\widehat{\psi}_{(n)}\right)\right)}\right)\right| \\
& \leqq k_{\varepsilon}\left|\widehat{\psi}-\widehat{\psi}_{(n)}\right| .
\end{aligned}
$$

Hence

$$
\left|\widehat{\psi}-\widehat{\psi}_{(n+1)}\right| \leqq k_{\varepsilon}^{n}\left|\widehat{\psi}-\widehat{\psi}_{(0)}\right|=k_{\varepsilon}^{n}|\widehat{\psi}-\psi| \leqq k_{\varepsilon}^{n}|\varpi| .
$$

This concludes the proof of Theorem 2.

\section{Application}

We illustrate, first, the theoretical results obtained for the local phase angle $\widehat{\psi}$ and then give some numerical comparisons with FrEED et al. [4] concerning phase transformation yield curves.

The latter is based on an "approximation" of Equation (41)

$$
r(\theta)=L \times \rho^{2} \times|\mathcal{G}(\boldsymbol{\Sigma}(\widehat{\psi}, \theta, \varepsilon, \bar{v}))|^{2} \forall \theta \in[0, \pi[,
$$

where the value of $\widetilde{\psi}$ in (41) (depending indirectly on $\theta$ ) has been fixed at $\widehat{\psi} \equiv$ $\widetilde{\psi}_{\mid \theta=0}$. The nearer to $r(0)$ the smaller the error. In particular, the error is zero when $\theta=0$ or $\varepsilon=0$.

In the sequel, we restrict ourselves to the cases $\xi=0$ and $\xi=1$ (see Remark 3) and use the following practical notations:

$$
\left\{\begin{array}{l}
\text { for } \xi=0, E_{1}=E_{A}: \rho_{s} \equiv \rho, \varepsilon_{A} \equiv \varepsilon, \psi_{s} \equiv \widehat{\psi}, r_{s} \equiv r \\
\text { for } \xi=1, E_{1}=E_{M}: \rho_{f} \equiv \rho, \varepsilon_{M} \equiv \varepsilon, \psi_{f} \equiv \widehat{\psi}, r_{f} \equiv r
\end{array},\right.
$$

where $\rho, \varepsilon, \widehat{\psi}$ and $r$ are determined respectively by (43), (6), (51) and (78).

\subsection{Physical Data}

Let us take the physical set (60) with $v=0.3$ and the Young's modulus values of the two materials

$\int$ Austenite Material (1): $E_{1}=E_{A}=70(\mathrm{GPa})$ Material (2) $: E_{2}=30(\mathrm{GPa})$ [Martensite Material (1) $: E_{1}=E_{M}=30(\mathrm{GPa})$ Material (2) $: E_{2}=30(\mathrm{GPa})$.

Let us choose as a shape function

$$
g(y)=\cos \left(\frac{1}{3} \arccos (1-a(1-y))\right), \quad \forall a \in[0,1] .
$$

The convexity proof of $\mathcal{G}$ can be found in LAYDI and LeXCELLENT [19]. 
Finally, we take the same values of $v, \rho_{s}, \widehat{L}$, and $L$ as in FreED et al. [4]

$$
\rho_{s}=2, \widehat{L}=5 \times 10^{-3}(\mathrm{~m}), L=4 \times 10^{-2}(\mathrm{~m}) .
$$

The $L$ value has not been given explicitly by FreED et al. [4]. This value is convenient for reproducing their numerical results in the particular case of symmetry between tension and compression, that is, $a=0$.

\subsection{Theoretical Bounds}

The $a$ value has no influence on $\psi_{f}$ because

$$
\varepsilon_{M}=0 \Rightarrow \psi_{f}(a)=\psi \forall a \in[0,1] .
$$

The situation is different for $\psi_{s}$, which depends on $a$, and by (53), we have the following bounds

$$
u=|\varepsilon| \ln \left(32 \times \delta^{2} \times|g(0)|^{2}\right) \leqq \psi-\psi_{s}(a) \leqq v=|\varepsilon| \ln \left(128 \times|g(0)|^{2}\right),
$$

with

$$
\varepsilon_{A} \simeq\left\{\begin{array}{l}
-0.045 \mathrm{CP} \\
-0.037 \mathrm{DP}
\end{array} \quad, \quad \delta^{2} \simeq\left\{\begin{array}{l}
0.45 \mathrm{CP} \\
0.1 \mathrm{DP}
\end{array}\right.\right.
$$

and

$$
g(0)=\cos \left(\frac{1}{3} \arccos (1-a)\right) .
$$

As $g(0) \in\left[\frac{\sqrt{3}}{2}, 1\right]$, one can deduce the following framing independent of $a$ :

$$
\left\{\begin{array}{l}
6.13^{\circ} \mathrm{CP} \\
1.85^{\circ} \mathrm{DP}
\end{array} \leqq \psi-\psi_{s}(a) \leqq\left\{\begin{array}{l}
12.47^{\circ} \mathrm{CP} \\
10.16^{\circ} \mathrm{DP}
\end{array} \quad \forall \psi, \forall a \in[0,1] .\right.\right.
$$

\subsection{Numerical Calculations}

According to the condition (57) $k_{\varepsilon}$ takes the numerical values

$$
k_{\varepsilon} \simeq\left\{\begin{array}{l}
0.27 \mathrm{CP} \\
0.46 \mathrm{DP}
\end{array}\right.
$$

These values are clearly less than the boundary $\frac{8}{3 \pi}$ coming from the theoretical estimation (61).

Hence, the numerical value $\widehat{\psi} \equiv \psi_{s}$ is unique. We obtain it by the algorithm (58) with the chosen stop criterion

$$
\left|\widehat{\psi}-\widehat{\psi}_{(n+1)}\right|<0.01^{\circ} \text {. }
$$

With this aim, it is sufficient to pose (see (59))

$$
2|\varepsilon| k_{\varepsilon}^{n} \ln \frac{2}{\delta}<0.01 \times \frac{\pi}{180},
$$




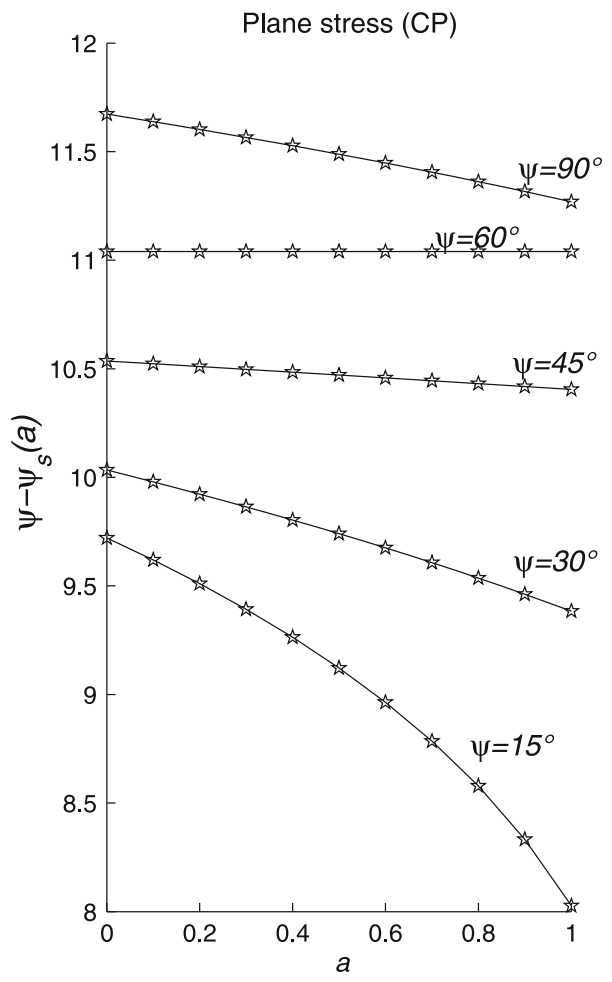

Fig. 2. Plane stress (CP). Effect of asymmetric parameter $a=\frac{i}{10}, i=0,1,2, \ldots, 10$, on the difference between applied phase angle and local phase angle

then

$$
n \geqq\left\{\begin{array}{l}
5 \mathrm{CP} \\
9 \mathrm{DP}
\end{array} .\right.
$$

The obtained numerical values $\psi_{s}$ are illustrated in Fig. 2 for plane stress and in Fig. 3 for plane strain conditions. The framing of $\left(\psi-\psi_{s}(a)\right)$ are also in agreement with the theoretical investigation (81).

Figure 2 for CP conditions and Fig. 3 for DP conditions show the evolution of the difference between the phase angle of the applied load and the local phase angle $\left(\psi-\psi_{s}(a)\right)$ as a function of the asymmetry parameter $a(a \in[0,1])$ with different phase angles of the applied load $\psi: 15^{\circ}, 30^{\circ}, 45^{\circ}, 60^{\circ}, 90^{\circ}$. The influence of $a$ on the value of $\left(\psi-\psi_{s}(a)\right.$ is more significant for the CP case than for the DP one, notably for small $\psi$. For example, the difference $\left(\psi_{s}(a=1)-\psi_{s}(a=0)\right)$ obtained for $\psi=15^{\circ}$ is equal to $1.7^{\circ}$ for $\mathrm{CP}$ and $0.2^{\circ}$ for DP.

\subsection{Phase Transformation Yield Curves}

The axes of the yield curves are, respectively,

$$
x=\frac{r}{6 L} \cos \theta \quad \text { and } \quad y=\frac{r}{6 L} \sin \theta, \theta \in[0, \pi],
$$




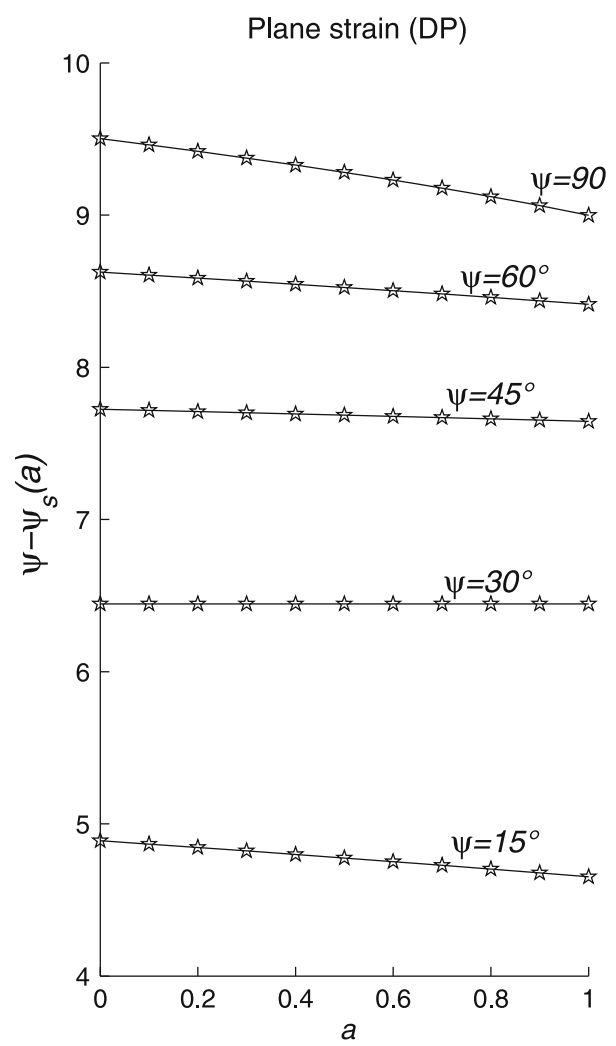

Fig. 3. Plane strain (DP). Effect of asymmetric parameter $a=\frac{i}{10}, i=0,1,2, \ldots, 10$, on the difference between applied phase angle and local phase angle

with the ratio $\frac{r}{L}$ according to expression (78), that is,

$$
\left\{\begin{array}{l}
r_{s}=L \times \rho_{s}^{2} \times\left|\mathcal{G}\left(\Sigma\left(\psi_{s}, \theta, \varepsilon_{A}, \bar{v}\right)\right)\right|^{2} \\
r_{f}=L \times \rho_{f}^{2} \times\left|\mathcal{G}\left(\Sigma\left(\psi_{f}, \theta, \varepsilon_{M}, \bar{v}\right)\right)\right|^{2}
\end{array}\right.
$$

Division by the number 6 has no particular meaning, but is useful to recreate the numerical results obtained by FREED et al. [4].

We choose to present the curves with $a=1$ because, in this case, the asymmetry effect is the most significant with regard to the symmetric case $a=0$.

Figure 4 (resp. 5) represents the transformation zones for plane strain conditions (resp. plane stress conditions) with $\psi=60^{\circ}, 45^{\circ}$ and $30^{\circ}$.

Observations of the different curves show that the width and the shape of the yield curves are nearly the same for symmetry $a=0$ and maximal asymmetry $a=1$. Moreover, we have performed a lot of simulations by taking different ratios of $E_{A}$ and $E_{M}$, without significant changes. A determination for pure mode I of the phase transformation zone at a crack tip of a single SMA plate shows that the $a$ value has an important influence on the width and shape of the yield curves (see LeXCELlent and Thiebaud [20]). Such is not the case here. 
(DP): $\psi_{f}=\psi=30^{\circ}$

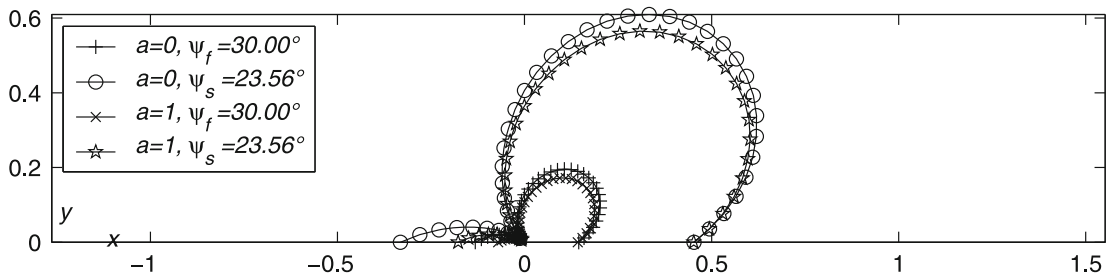

(DP): $\psi_{f}=\psi=45^{\circ}$

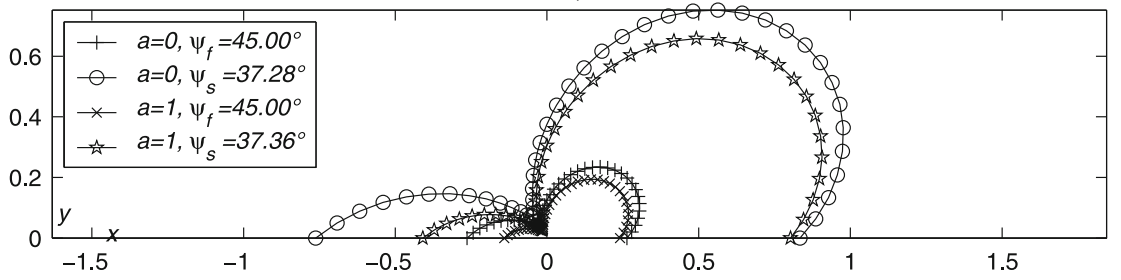

(DP): $\psi_{f}=\psi=60^{\circ}$

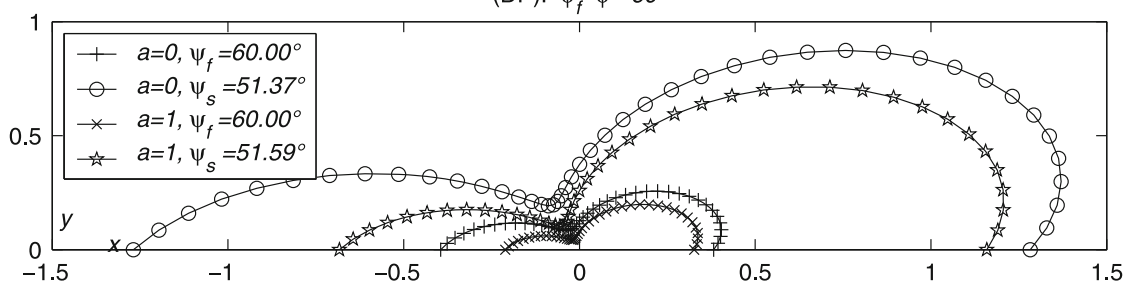

Fig. 4. Transformation zones for plane strain conditions with $\psi=60^{\circ}, 45^{\circ}$ and $30^{\circ}$

\section{Remarks and Conclusion}

Note that we have chosen the same material data as FREED et al. [4] in order to extend the SMA modeling by integrating the asymmetry between tension and compression in the yield curve predictions. Naturally the Rice phase angle $\widehat{\psi}$ will change if one modifies certain parameters of the materials following (52).

One can choose a shape function $g$ other than the one of (79). The difference can be estimated according to (62), that is,

$$
2 \varepsilon \ln \left(\frac{2}{\delta} \frac{g_{1}(0)}{g_{2}(0)}\right) \leqq \widehat{\psi}^{\left(g_{1}\right)}-\widehat{\psi}^{\left(g_{2}\right)} \leqq 2 \varepsilon \ln \left(\frac{1}{2} \delta \frac{g_{1}(0)}{g_{2}(0)}\right) \forall \varepsilon \leqq 0 .
$$

For example (see (79)), by choosing $g_{1} \equiv g$ (with $\left.a=0.5\right)$ and $g_{2} \equiv g$ (with $a=0$ ), that is,

$$
g_{1}(y)=\cos \left(\frac{1}{3} \arccos \left(1-\frac{1}{2}(1-y)\right)\right) \text { and } g_{2}(y)=1,
$$

one finds

$$
\left\{\begin{array}{l}
-5.31^{\circ} \mathrm{CP} \\
-8.1^{\circ} \mathrm{DP}
\end{array} \leqq \widehat{\psi}^{\left(g_{1}\right)}-\widehat{\psi}^{\left(g_{2}\right)} \leqq\left\{\begin{array}{l}
5.96^{\circ} \mathrm{CP} \\
8.6^{\circ} \mathrm{DP}
\end{array} \forall \psi\right.\right.
$$

These bounds are in perfect agreement with Fig. 2 for CP and Fig. 3 for DP. 


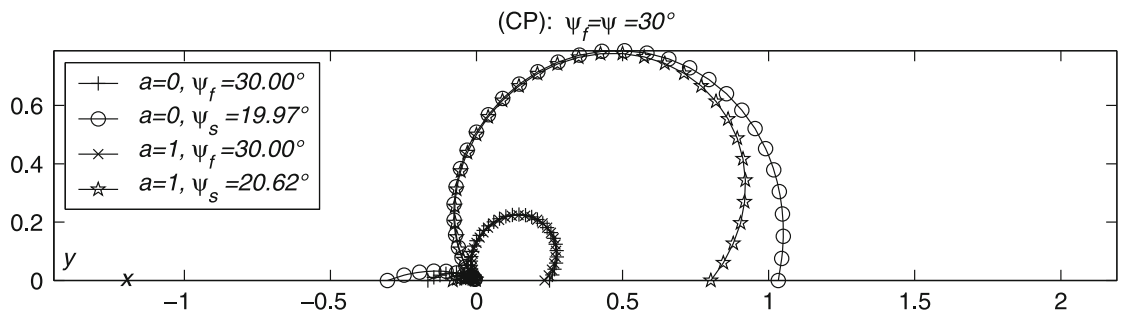

(CP): $\psi_{f}=\psi=45^{\circ}$

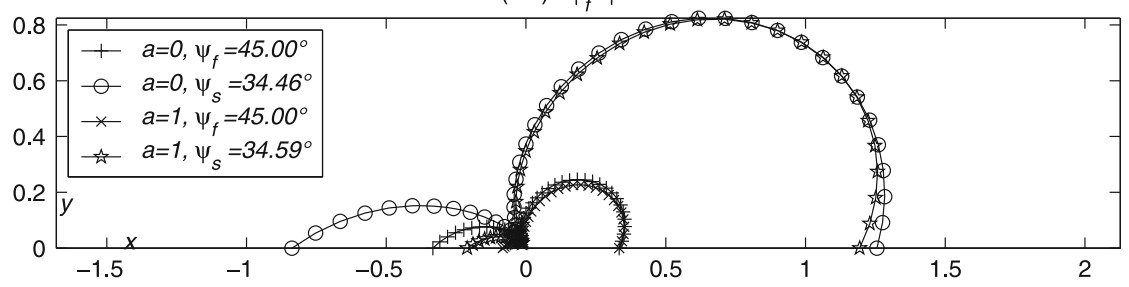

(CP): $\psi_{f}=\psi=60^{\circ}$

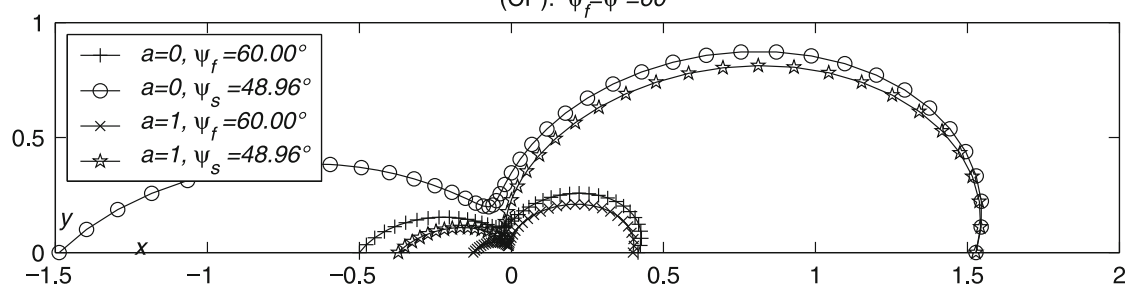

Fig. 5. Transformation zones for plane stress conditions with $\psi=60^{\circ}, 45^{\circ}$ and $30^{\circ}$

A second example can be

$$
g_{1}(y)=1+b y\left(\forall b \in\left[0, \frac{1}{8}\right]\right) \quad \text { and } \quad g_{2}(y)=1
$$

where one finds

$$
\left\{\begin{array}{l}
-5.64^{\circ} \mathrm{CP} \\
-7.83^{\circ} \mathrm{DP}
\end{array} \leqq \widehat{\psi}^{\left(g_{1}\right)}-\widehat{\psi}^{\left(g_{2}\right)} \leqq\left\{\begin{array}{l}
5.64^{\circ} \mathrm{CP} \\
7.83^{\circ} \mathrm{DP}
\end{array} \forall \psi\right.\right.
$$

Actually, delamination experiments are in progress. Particular attention is paid to the measurement of the displacement field around the crack tip. Moreover, an infrared camera allows us to estimate the temperature field in order to detect the transformation zones because the phase transformation is exothermal.

A sequel paper will be devoted to the comparison between theoretical predictions and experimental results.

Acknowledgments We would like to thank NamaH G. for the interest devoted to this paper. 


\section{Appendices}

\subsection{Appendix A}

Recall that a stress tensor $\boldsymbol{\Sigma}$ in a cartesian coordinate system is given by $\boldsymbol{\Sigma}_{(r \theta z)}$ in the cylindrical coordinate system by

$$
\Sigma_{(r \theta z)}=\mathcal{R}^{T} \boldsymbol{\Sigma} \mathcal{R}
$$

where

$$
\mathcal{R} \equiv\left(\begin{array}{lll}
\cos (\theta) & -\sin (\theta) & 0 \\
\sin (\theta) & \cos (\theta) & 0 \\
0 & 0 & 1
\end{array}\right)
$$

Thus, one checks that tensors associated to (26) are given the cylindrical coordinate system by

$$
\Sigma^{I} \equiv\left(\begin{array}{lll}
\Sigma_{r r}^{I} & \Sigma_{r \theta}^{I} & 0 \\
\Sigma_{r \theta}^{I} & \Sigma_{\theta \theta}^{I} & 0 \\
0 & 0 & \bar{v}\left(\Sigma_{r r}^{I}+\Sigma_{\theta \theta}^{I}\right)
\end{array}\right) ; \quad \Sigma^{I I} \equiv\left(\begin{array}{lll}
\Sigma_{r r}^{I I} & \Sigma_{r \theta}^{I I} & 0 \\
\Sigma_{r \theta}^{I I} & \Sigma_{\theta \theta}^{I I} & 0 \\
0 & 0 & \bar{v}\left(\Sigma_{r r}^{I I}+\Sigma_{\theta \theta}^{I I}\right)
\end{array}\right)
$$

such that $\left(\pi_{\alpha} \equiv(-1)^{\alpha} \pi\right)$

$$
\left\{\begin{array}{l}
\Sigma_{r r}^{I}=\frac{\sinh \left(\varepsilon\left(\pi_{\alpha}+\theta\right)\right)}{\cosh (\pi \varepsilon)} \cos \left(\frac{3 \theta}{2}\right)+\frac{\exp \left(\varepsilon\left(\pi_{\alpha}+\theta\right)\right)}{\cosh (\pi \varepsilon)} \cos \left(\frac{\theta}{2}\right)\left(1+\sin ^{2}\left(\frac{\theta}{2}\right)+\varepsilon \sin (\theta)\right) \\
\Sigma_{\theta \theta}^{I}=-\frac{\sinh \left(\varepsilon\left(\pi_{\alpha}+\theta\right)\right)}{\cosh (\pi \varepsilon)} \cos \left(\frac{3 \theta}{2}\right)+\frac{\exp \left(\varepsilon\left(\pi_{\alpha}+\theta\right)\right)}{\cosh (\pi \varepsilon)} \cos \left(\frac{\theta}{2}\right)\left(\cos ^{2}\left(\frac{\theta}{2}\right)-\varepsilon \sin (\theta)\right) \\
\boldsymbol{\Sigma}_{r \theta}^{I}=-\frac{\sinh \left(\varepsilon\left(\pi_{\alpha}+\theta\right)\right)}{\cosh (\pi \varepsilon)} \sin \left(\frac{3 \theta}{2}\right)+\frac{\exp \left(\varepsilon\left(\pi_{\alpha}+\theta\right)\right)}{\cosh (\pi \varepsilon)} \sin \left(\frac{\theta}{2}\right)\left(\cos ^{2}\left(\frac{\theta}{2}\right)-\varepsilon \sin (\theta)\right) \\
\boldsymbol{\Sigma}_{r r}^{I I}=\frac{\cosh \left(\varepsilon\left(\pi_{\alpha}+\theta\right)\right)}{\cosh (\pi \varepsilon)} \sin \left(\frac{3 \theta}{2}\right)-\frac{\exp \left(\varepsilon\left(\pi_{\alpha}+\theta\right)\right)}{\cosh (\pi \varepsilon)} \sin \left(\frac{\theta}{2}\right)\left(\left(1+\cos ^{2}\left(\frac{\theta}{2}\right)-\varepsilon \sin (\theta)\right)\right. \\
\boldsymbol{\Sigma}_{\theta \theta}^{I I}=-\frac{\cosh \left(\varepsilon\left(\pi_{\alpha}+\theta\right)\right)}{\cosh (\pi \varepsilon)} \sin \left(\frac{3 \theta}{2}\right)-\frac{\exp \left(\varepsilon\left(\pi_{\alpha}+\theta\right)\right)}{\cosh (\pi \varepsilon)} \sin \left(\frac{\theta}{2}\right)\left(\sin ^{2}\left(\frac{\theta}{2}\right)+\varepsilon \sin (\theta)\right) \\
\boldsymbol{\Sigma}_{r \theta}^{I I}=\frac{\cosh \left(\varepsilon\left(\pi_{\alpha}+\theta\right)\right)}{\cosh (\pi \varepsilon)} \cos \left(\frac{3 \theta}{2}\right)+\frac{\exp \left(\varepsilon\left(\pi_{\alpha}+\theta\right)\right)}{\cosh (\pi \varepsilon)} \cos \left(\frac{\theta}{2}\right)\left(\sin ^{2}\left(\frac{\theta}{2}\right)+\varepsilon \sin (\theta)\right)
\end{array} .\right.
$$

We find, again, the explicit expression of the stress tensor already given by RICE [1].

\subsection{Appendix $B$}

Lemma 2. Let $\beta$ be given by (7), (8) and (9). Then (11) is satisfied and (12) holds for $v_{1}=v_{2}=v$.

Proof. The following expression of $\beta$ in terms of $e \equiv \frac{E_{2}}{E_{1}}$ and of $v_{i}$ results from the formulae (7)-(9). One gets

$$
2 \beta=\left\{\begin{array}{ll}
\frac{\left(1-v_{2}\right)-\left(1-v_{1}\right) e}{1+e} & \text { for CP } \\
\frac{p\left(v_{2}\right)\left(1-v_{2}^{2}\right)-p\left(v_{1}\right)\left(1-v_{1}^{2}\right) e}{\left(1-v_{2}^{2}\right)+\left(1-v_{1}^{2}\right) e} & \text { for DP }
\end{array},\right.
$$

with $e>0$ and where

$$
p: v \in] 0, \frac{1}{2}\left[\rightarrow p(v) \equiv \frac{1-2 v}{1-v} .\right.
$$


Thus for the CP case, we have

$$
2|\beta| \leqq \max \left\{1-v_{2}, 1-v_{1}\right\} \leqq 1-v<1,
$$

with

$$
v=\min \left\{v_{1}, v_{2}\right\}
$$

and for DP, as $p$ is decreasing with positive values, one also gets

$$
2|\beta| \leqq \max \left\{p\left(v_{2}\right), p\left(v_{1}\right)\right\} \leqq p(v)<1-v<1 .
$$

Finally, we always have

$$
|\beta| \leqq \widetilde{\beta} \equiv \frac{1}{2}\left\{\begin{array}{l}
1-v \text { for } \mathrm{CP} \\
p(v) \text { for DP }
\end{array}<\frac{1}{2} \text { where } v=\min \left\{v_{1}, v_{2}\right\} .\right.
$$

This ends the proof of (11).

The expression of $\beta$ (see (12)) is a direct consequence of (87).

\subsection{Appendix $C$}

Proof (61). The case $e \equiv \frac{E_{2}}{E_{1}}=1$ is trivial because $\varepsilon=0$ (see (12)). Let then $e<1$. Due to assumption (60), we find ourselves in the case where (see (12))

$$
0<\beta \leqq \widetilde{\beta} \equiv \frac{1}{2}\left\{\begin{array}{l}
1-v \text { for CP } \\
p(v) \text { for DP }
\end{array}, p(v) \equiv \frac{1-2 v}{1-v}, v \in\right] 0, \frac{1}{2}[
$$

We are going to show that

$$
\widetilde{k}_{\varepsilon} \equiv \frac{3 \pi}{8} \underbrace{k_{\varepsilon}}_{\frac{4}{\delta}|\varepsilon|}=\frac{3 \pi}{8} \frac{4}{\delta} \frac{\operatorname{arctanh}(\beta)}{\pi}=\frac{3}{2} \frac{\operatorname{arctanh}(\beta)}{\delta}<1 .
$$

By Taylor's formulae, we also have

$$
\widetilde{k}_{\varepsilon} \leqq \frac{3}{2} \frac{\beta}{\delta} \frac{1}{1-\beta^{2}} \leqq \frac{3}{2} \frac{\beta}{\delta} \frac{1}{1-\widetilde{\beta}^{2}} .
$$

Recall that

$$
\frac{\beta}{\delta}=\frac{1}{\sqrt{1+\frac{1}{3}\left(1+\frac{1-2 \bar{v}}{\beta}\right)^{2}}}
$$

The CP case is immediate; on the one hand

$$
\frac{1-2 \bar{v}}{\beta}=\frac{1}{\beta}>2 \Rightarrow \frac{\beta}{\delta}<\frac{1}{\sqrt{1+\frac{1}{3}(1+2)^{2}}}=\frac{1}{2},
$$


and on the other hand

$$
\frac{1}{1-\widetilde{\beta}^{2}}<\frac{1}{1-\left(\frac{1}{2}\right)^{2}}=\frac{4}{3}
$$

For the DP case, we have

$$
\begin{aligned}
\frac{1-2 \bar{v}}{\beta} & >\frac{1-2 v}{\widetilde{\beta}}=2(1-v)=\frac{2}{2-p} \Rightarrow \frac{\beta}{\delta}<\frac{1}{\sqrt{1+\frac{1}{3}\left(1+\frac{2}{2-p}\right)^{2}}} \\
& =\frac{\sqrt{3}}{2} \frac{2-p}{\sqrt{p^{2}-5 p+7}}
\end{aligned}
$$

and

$$
\frac{1}{1-\widetilde{\beta}^{2}}=\frac{4}{4-p^{2}}
$$

Therefore

$$
\tilde{k}_{\varepsilon}<\frac{3}{2} \frac{\sqrt{3}}{2} \frac{2-p}{\sqrt{p^{2}-5 p+7}} \frac{4}{4-p^{2}}=\sqrt{\frac{27}{f(p)}},
$$

where

$$
f(p)=(2+p)^{2}\left(p^{2}-5 p+7\right) .
$$

But as

$$
f(p)=(p(1-p)+1)\left(7+\left(1-p^{2}\right)\right)+20 \geqq 27 \forall p \in[0,1],
$$

the result (61) follows.

\section{References}

1. Rice, J.R., Suo, Z., WANG, J.S. : Mechanics and thermodynamics of brittle interfacial failure in bimaterial systems. In: Ruhle, M., Evans, A.C., Ashby, M.F., Hirth, J.P. (eds.) Metal Ceramic Interfaces. Pergamon Press, New York, 269-294, 1990

2. YI, S., GAO, S.: Fracture toughening mechanism of shape memory alloys due to martensite transformation. Int. J. Solids Struct. 37(38), 5315-5327 (2000)

3. FreEd, L., BANKS-SiLl, L.: Crack growth resistance of shape memory alloys by means of a cohesive zone model. J. Mech. Phys. Solids 55(10) 2157-2180 (2007)

4. Freed, L., BANKs-Sill, L., ABoudi, J.: On the transformation toughening of a crack along an interface between a shape memory alloy and an isotropic medium. J. Mech. Phys. Solids 56, 3003-3020 (2008)

5. Panoskaltis, V.P., Bahugana, S., Soldatos, D.: On the thermomechanical modeling of shape memory alloys. Int. J. Non-Linear Mech. 39(5), 709-722 (2004)

6. VACHer, P., LeXCEllent, C.: Study of pseudoelastic behavior of polycrystalline SMA by resistivity measurements and acoustic emission. Proc ICM 6, 231-236 (1991)

7. Orgéas, L., FAvier, D.: Stress-induced martensitic transformation of a Ni-Ti alloy in isothermal shear, tension and compression. Acta Mater. 46(15), 5579-5591 (2000) 
8. Suo, Z.: Singularities acting with interfaces and cracks. Int. J. Solids Struct. 25(10), 1133-1142 (1989)

9. Daly, S., Miller, A., Ravichandran, G., Bhattacharya, K.: Experimental investigation of crack initiation in thin sheets of nitinol. Acta Mater. 55(18), 6322-6330 (2007)

10. Rice, J.R., Parks, D.M., Sorensen, E.P.: Recent finite element studies in plasticity and fracture mechanics. Comput. Methods Appl. Mech. Eng. 17/18, 411-442 (1979)

11. Dundurs, J.: Some properties of elastic stresses in a composite. In: Eringen, A.C. (ed.) Recent Advances in Engineering Science, vol. 5. Gordon and Breach Science Publishers, New York, 203-216, 1970

12. Lexcellent, C., Boubakar, L., Bouvet, C., Calloch, S.: About modelling the shape memory alloy behaviour based on the phase transformation surface identification under proportional loading and anisothermal conditions. Int. J. Solids Struct. 43(3-4), 613-626 (2006)

13. Bernardini, D., Pence, T.J.: Shape memory alloys: modeling. In: Encyclopedia of Smart Materials, vol. 2. Wiley, New York, 964-980, 2002

14. Evans, A. G.: Toughening mechanisms in zirconia alloys. In: Claussen, M., Ruhle, M., Heuer, A.H. (eds.) Science and Technology of Zirconia II. American Ceramic Society, Columbus, 193-212, 1984

15. Rice, J. R.: Elastic fracture mechanics concepts for interfacial cracks. J. Appl. Mech. 55, 98-103 (1988)

16. Raniecki, B., Lexcellent, C.: Thermodynamics of isotropic pseudoelasticity in shape memory alloys. Eur. J. Mech. A/Solids 17(2), 185-205 (1998)

17. Bouvet, C., Calloch, S., Lexcellent, C.: A phenomenological model for pseudoelasticity of shape memory alloys under multiaxial proportional and nonproportional loading. Eur. J. Mech. A/Solids 23, 37-61 (2004)

18. Hill, R.: The Mathematical Theory of Plasticity. Clarendon Press, Oxford, 1950

19. LAYDI, M.R., LEXCELlENT, C.: Yield criteria for shape memory materials: convexity conditions and surface transport. Math. Mech. Solids 15(2), 165-208 (2010)

20. Lexcellent, C., Thiebaud, F.: Determination of the phase transformation zone at a crack tip in a shape memory alloy exhibiting asymmetry between tension and compression. Scripta Mater. 59(3), 321-323 (2008)

FEMTO-ST Département de Mécanique Appliquée, 24 rue de l'épitaphe 25000 Besançon, France. e-mail: rachid.laydi@ens2m.fr e-mail: christian.lexcellent@univfcomte.fr 